\title{
Insights into N6-methyladenosine and programmed cell death in cancer
}

\author{
Li Liu ${ }^{1,2+}$, Hui Li ${ }^{3,4 \dagger}$, Dingyu Hu², Yanyan Wang ${ }^{2}$, Wenjun Shao ${ }^{2}$, Jing Zhong ${ }^{5}$, Shudong Yang ${ }^{1}$, Jing Liu ${ }^{3^{*}}$ and \\ Ji Zhang ${ }^{1,2^{*}}$ (D)
}

\begin{abstract}
N6-methyladenosine (m6A) methylation, the most common form of internal RNA modification in eukaryotes, has gained increasing attention and become a hot research topic in recent years. M6A plays multifunctional roles in normal and abnormal biological processes, and its role may vary greatly depending on the position of the m6A motif. Programmed cell death (PCD) includes apoptosis, autophagy, pyroptosis, necroptosis and ferroptosis, most of which involve the breakdown of the plasma membrane. Based on the implications of m6A methylation on PCD, the regulators and functional roles of m6A methylation were comprehensively studied and reported. In this review, we focus on the high-complexity links between m6A and different types of PCD pathways, which are then closely associated with the initiation, progression and resistance of cancer. Herein, clarifying the relationship between m6A and PCD is of great significance to provide novel strategies for cancer treatment, and has a great potential prospect of clinical application.
\end{abstract}

Keywords: N6-methyladenosine, Cancer, Apoptosis, Autophagy, Pyroptosis, Ferroptosis, Necroptosis

\section{Introduction}

To date, out of more than 160 chemical modifications, N6-methyladenosine, a methylation modification of the sixth adenine (A) nitrogen, is the most common internal RNA modification occurring at the post-transcriptional level, especially in eukaryotic mRNAs [1-4]. M6A methylation frequently occurs around stop codons and $3^{\prime}$ untranslated regions ( $3^{\prime}$-UTRs) and is modulated by three regulators: writers, readers and erasers [5], subsequently affecting related physiological functions and

\footnotetext{
*Correspondence: jingliucsu@hotmail.com; zhang_ji001@hotmail.com ${ }^{\dagger}$ Li Liu and Hui Li contributed equally to this work.

${ }^{2}$ The First Affiliated Hospital, Department of Rheumatology, Hengyang Medical School, University of South China, Hengyang 421001, Hunan, China

${ }^{3}$ Hunan Province Key Laboratory of Basic and Applied Hematology, Molecular Biology Research Center \& Center for Medical Genetics, School of Life Sciences, Central South University, Changsha 410078, Hunan, China

Full list of author information is available at the end of the article
}

pathological processes, particularly in tumorigenesis [6-8].

In most cases, m6A plays oncogenic roles in the tumorigenesis and development of cancers, such as gastric cancer (GC), bladder cancer and hepatocellular carcinoma (HCC) [9-12]. However, it also has been reported that inhibiting METTL14 (methyltransferase-like protein 14)-mediated m6A abundance facilitates the development of bladder tumor-initiating cells (TICs) [13]. Whether m6A methylations exhibit anticancer or protumoral effects remain controversial, which may depend on the various characteristics of cancer, such as the intratumoral microenvironment $[14,15]$. As more attention has been given to its role in the tumor process, m6A methylations have been increasingly investigated in ovarian cancer [16], colorectal cancer [17], breast cancer [18], and so on. Moreover, several lines of evidence have revealed the essential roles played by m6A RNA modifications (i.e., m6A epitranscriptome) in drug resistance [19], NAFLD development [20, 21], type 2 diabetes [22] 
and the occurrence of viral hepatitis [23-25], as well as malignant hematopoiesis [26].

Emerging evidence suggests that the combination of apoptosis with other forms of programmed cell death, such as autophagy, pyroptosis, necroptosis, NETosis and ferroptosis, is significantly required for the maintenance of normal cell cycle and tissue homeostasis, and responding in an actively regulated manner to either internal or external threats [27-32]. Programmed cell death (PCD) played a fundamental role in an organism's balance mechanism by which stressed, damaged, malignant or infected cells were lysed and effectively cleared [33, 34]. Studies have shown that distinct types of cell death are not independent of each other and share a coordinated system. When one pathway is abnormally defective, other regulatory mechanisms will ensure the process of cell death. Importantly, malfunctioning PCD pathways are related to the occurrence and development of diseases [33-35].

As the most prevalent epigenetic modification on mRNAs and noncoding RNAs, m6A methylation influences RNA metabolism, thus negatively controlling protein expression in a posttranscriptional manner; moreover, PCD elicits cell death via complicated cascades of transcriptional alterations and posttranslational protein modifications [32, 36]. Over the past years, mounting evidence has proven that $\mathrm{m} 6 \mathrm{~A}$ may regulate gene expression, thus affecting diverse programmed cell death processes.

\section{Methods for m6A analysis}

After the discovery of the restriction-modification (R-M) system in the 1960s, m6A marks were first identified in bacterial DNA and extensively studied in eukaryotic DNA and RNA [37-40], which further promoted the related research on m6A in RNAs. However, due to the lack of available methods to define its position in RNA, the biological function of $\mathrm{m} 6 \mathrm{~A}$ remained largely elusive. It was not until 2011 that the identification of demethylase, fat mass and obesity-associated protein (FTO) directly promoted the development of m6A analysis methods, which established the view of m6A as a dynamic and highly conserved post-transcriptional modification that contains a consensus motif termed "RRACH" in methylatedm6A RNAs [41-44]. To date, many new m6A-related technologies have been developed. For example, MeRIPm6A-seq allows a global view of ubiquitous RNA peaks as the first protocol. This technique pulls down m6Acontaining RNA fragments through specific anti-m6A antibody. Most of the m6A sites in the $3^{\prime}$ - UTR from the human transcriptome have been identified. However, the size of the RNA fragment pulled down leads to limited resolution [41, 45], photo-cross-linking-assisted m6A sequencing (PA-m6A-seq) combines antibody-based immunoprecipitation to improve the resolution of $\mathrm{m} 6 \mathrm{~A}$ profiling, which realizes further breakthroughs in locating m6A sites [46], and matrix-assisted laser desorption/ ionization time of flight mass spectrometry (MALDITOF-MS) purposefully detects m6A mutations in the corresponding miRNAs. This method alkylates the N1 of adenines in RNA with dimethylsulfate followed by sequential MS analysis [47]. Due to the quickly evolving m6A detection method, the panorama of $\mathrm{m} 6 \mathrm{~A}$ in cancer is expected to apply to cancer diagnosis and molecular classifications in the future.

\section{$M 6 A$ regulators}

M6A writers, readers and erasers are involved in the initiation, recognition and removal of coding and noncoding RNAs (ncRNAs), respectively (Fig. 1). The detailed regulation of $\mathrm{m} 6 \mathrm{~A}$ is as follows: $\mathrm{m6A}$ writers (METTL3, METTL14, METTL16, WTAP, RBM15/15B, VIRMA, ZC3H13) install m6A sites in RNAs, paramountly, forming a methyltransferase complex (MTC) between methyltransferase-like protein 3 (METTL3) and METTL14. The former mainly catalyzes the transfer of methyl groups in S-adenosyl methionine (SAM) to adenine bases in RNA, whereas the latter stabilizes the conformation of MTC and appraises precise RNA sequences, with additional subunits contributing to the activity and specificity of this complex [48-51]. Wilms tumor 1-associating protein (WTAP) recruits METTL3 and METTL14 into nuclear speckles, promoting RNA-binding capability of $\mathrm{m} 6 \mathrm{~A}$ methyltransferase. WTAP and METTL3 regulate various genes related to transcription and RNA processing [52]; RNA binding motif protein 15/15B(RBM15/15B) directs METTL3-METTL14 heterodimer to specific RNA sites [53, 54]; Vir like m6A methyltransferase associated (VIRMA) is reported to recruit the MTC and interact with polyadenylation cleavage factors CPSF5 and CPSF6 [55]; Zinc finger CCCH-type containing 13(ZC3H13) keeps MTC location in a WTAP-binding fashion [56]; In comparison with MTC, METTL16 preferentially mediates m6A epitranscriptomic modification of U6-snRNA in a divergent sequence and structure setting [57].

In response to external forces, $\mathrm{m} 6 \mathrm{~A}$ is identified by diverse readers (YTH domain family, IGF2BPs, HNRNP family, eIF3), triggering a series of downstream biological responses. YTH domain containing 1(YTHDC1) promotes RNA processing and export [58-60]; YTHDC2 enhances target mRNA translation incidence through reducing its abundance [61, 62]; YTH domain family protein 1 (YTHDF1) acts as a translation promoter while YTHDF2 degradation accelerates the downregulation of mRNA levels, and YTHDF3 coordinates with these proteins synergistically, playing an assistant role 


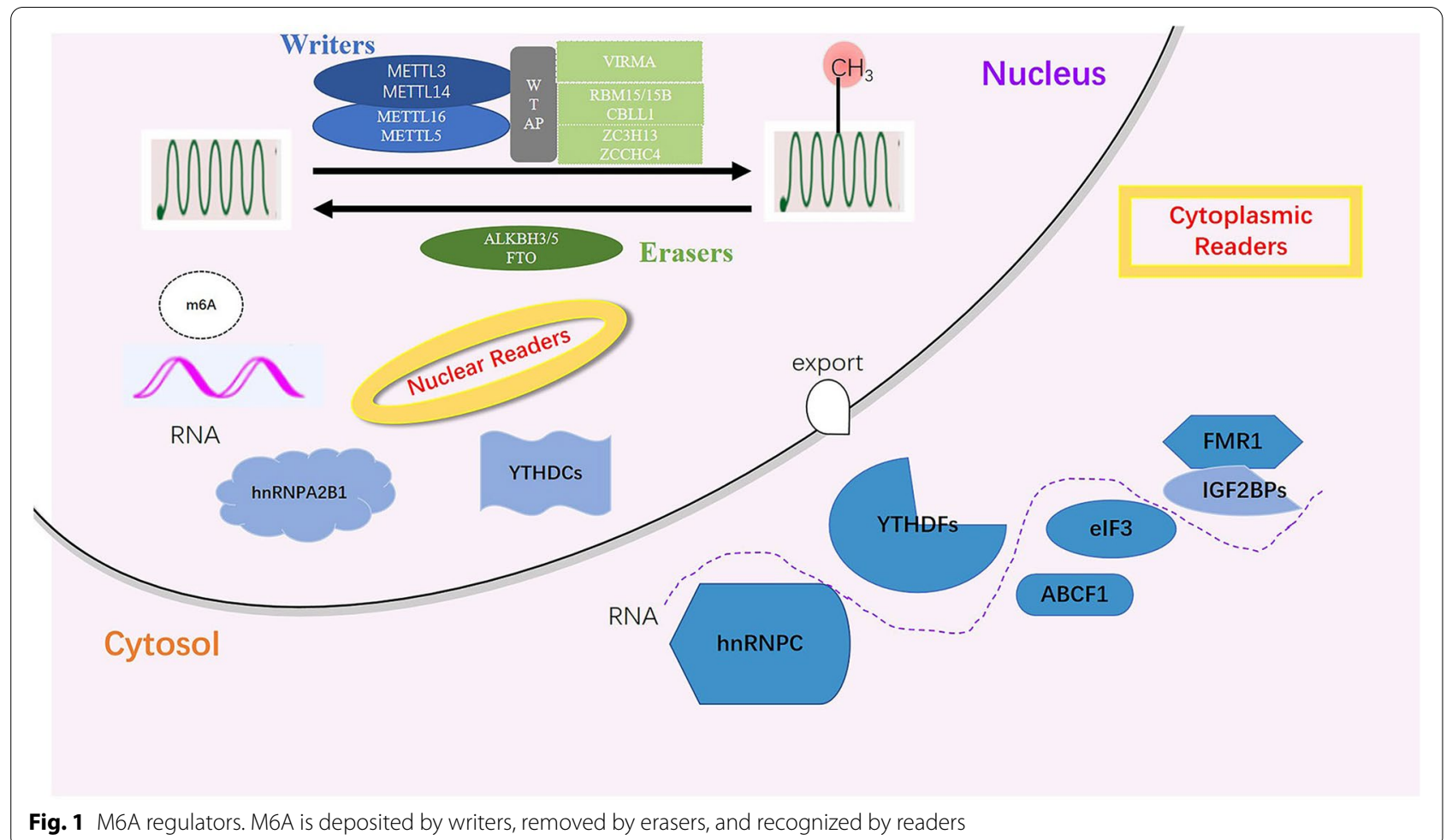

in target mRNA [63-66]. Insulin-like growth factor 2 mRNA-binding proteins (IGF2BP1/2/3) stabilizes mRNA and promotes translation with eukaryotic translation initiation factor 3 (eIF3) [67, 68]. Heterogeneous nuclear ribonucleoprotein A2/B1 (HNRNPA2/B1) facilitates pri-miRNA processing [69]. In addition, HNRNPC/G interacts with m6A-modified mRNA and affects its enrichment and splicing, generating a phenomenon termed the "m6A switch" [70, 71]. Finally, m6A erasers, FTO and ALKBH3/5, reverse m6A and promote RNA demethylation $[72,73]$. These results have revealed how these enzymes and binding proteins modulate m6Amethylated RNAs. It is noted that these "effectors" are not independent components. Furthermore, homeostasis of m6A chemical sites on RNAs is retained in a contextreliant manner, thus impacting gene expression [74].

\section{M6A modifications in RNA metabolism}

As mentioned above, m6A methylations were involved in mRNA metabolism by an expanding list of $\mathrm{m} 6 \mathrm{~A}$ readers and $\mathrm{m} 6 \mathrm{~A}$ writer-complex components, as well as erasers, exerting effects on mRNA splicing, translation, and decay [75]. Notably, as described by Berulava et al., transcripts mainly carry m6A modifications around the translation end sites, $5^{\prime}$-UTR or $3^{\prime}$-UTR and coding sequences (CDS). Depending on its location, m6A methylation sites might determine the functional consequences, leading to intricate influences on the mRNA. Specifically, m6A-containing transcripts in $5^{\prime}$-UTR and/or CDS are associated with energy metabolism, mitochondrial function and intracellular pathways, while the majority of transcripts methylated in the $3^{\prime}$-UTR are linked to more specific metabolic processes, including "acetyl-CoA or glycerol biosynthesis" and "positive regulation of protein dephosphorylation". However, the detailed mechanism remains elusive [76].

Accumulating evidences have shown that m6A decorations also account for indispensable parts in ncRNA processing [77]. The effects of m6A on miRNAs are mediated by a series of $\mathrm{m} 6 \mathrm{~A}$ regulatory proteins. For example, steady-state levels of several miRNAs, such as let-7e, miR-25, miR-93, miR-126, miR-221/222, and miR-4485, could be affected by altered METTL3, thus facilitating miRNA biogenesis [78]. Similar to METTL3, METTL14, another m6A writer, can also govern the expression of miRNAs with m6A peaks [79]. In addition to the two core compositions of MTC, m6A readers participate in miRNA biogenesis and maturation, especially HNRNPA2B1 [69, 80-82]. The FTO and NOP2/Sun domain family (NSun2, a methyltransferase) play an inhibitory role in m6A-mediated miRNA regulation [83, 84]. Additionally, previous studies indicated the role of m6A readers on circRNAs [85-88]. In addition, m6A is actively involved in the interactions between lncRNAs and other 
RNAs, such as miRNAs [89], controlling many aspects of gene expression and cellular biology. M6A-snRNAs catalyze pre-mRNA splicing and biogenesis, while m6ArRNAs manipulate target transcript translation [90, 91], representing typical cases of ncRNA-mRNA interactions. Accordingly, m6A is involved not only in mRNA biogenesis and functions but also in miRNA maturation, circRNA translation, and RNA-RNA interplay. Collectively, the multiple biofunctions of RNAs that are highly m6Amodified, many researchers have reached a consensus on whether and how ncRNAs or mRNA are greatly affected by $\mathrm{m} 6 \mathrm{~A}$ regulators (Fig. 2). However, the effects should have been virtually made to investigate the specific proofof-principle of m6A depositions on mRNA and the role of $\mathrm{m} 6 \mathrm{~A}$ regulators in the transcriptome of ncRNAs.

\section{M6A and programmed cell death}

Since it was first identified in apoptosis through modulating gene expression, m6A posed by an increasing number of studies is interplayed with almost all types of PCD pathways. Here, we mainly discuss the relevance between m6A and cell apoptosis, autophagy, pyroptosis, and ferroptosis, as well as necroptosis (Fig. 3). There is no doubt that association of $\mathrm{m} 6 \mathrm{~A}$ and $\mathrm{PCD}$ pathways will provide new insights into the management of related diseases. It is worth mentioning that the interconnectivity among various PCD types significantly dictates cell fate. For instance, autophagy can induce necroptosis.

\section{M6A and apoptosis}

Numerous studies have found biomarker value in the relevance of m6A and apoptosis. As summarized by Chen et al., m6A induces apoptosis via four mechanisms: governing apoptosis-related genes, silencing methylating or demethylating enzyme genes, and reducing transcripts mediated by Ythdf2 [92]. More reader proteins have been considered activators resulting in apoptosis based on recent reports. According to machine learning and GSEA analysis, the YTHDC2 gene, ranked the highest among 10 genes in experimental models, may increase the translation efficiency of Smc3 (target YTHDC2 in spermatogenesis) and stimulate the significant elevation of apoptosis-associated genes sush as caspase 3, Bcl-2, and Bid in neck squamous cancer cells, implying better overall survival (OS) in contrast to other regulatory genes [93]. However, the underlying mechanism has not yet been elucidated. In a recent study investigating lncRNAs harboring m6A, LNC942 directly recruited METTL14, a core member of m6A methyltransferase complexes, accompanied by elevated epigenetic m6A methylation modification levels in breast cancer cells, subsequently accelerating cell proliferation and colony formation and decreasing cellular

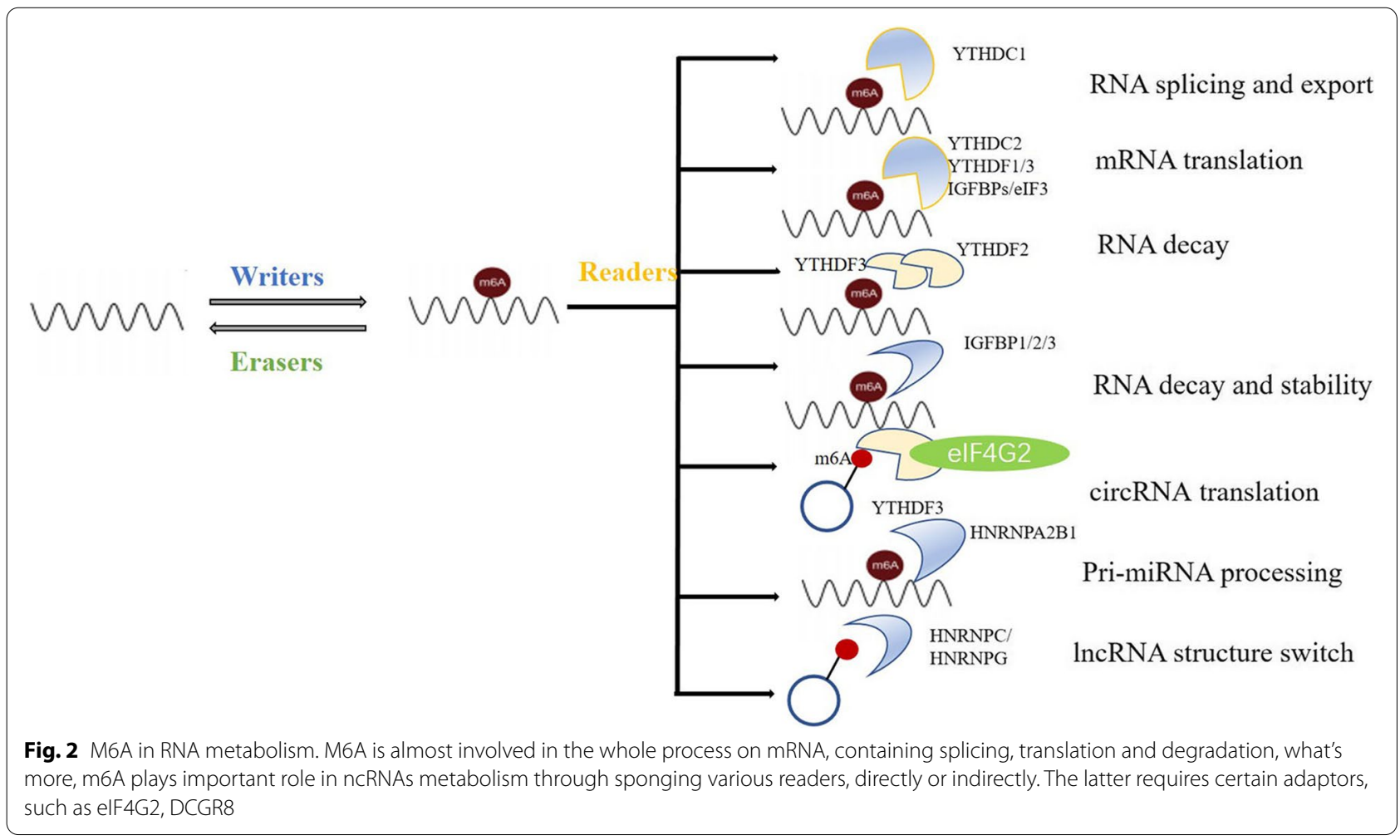




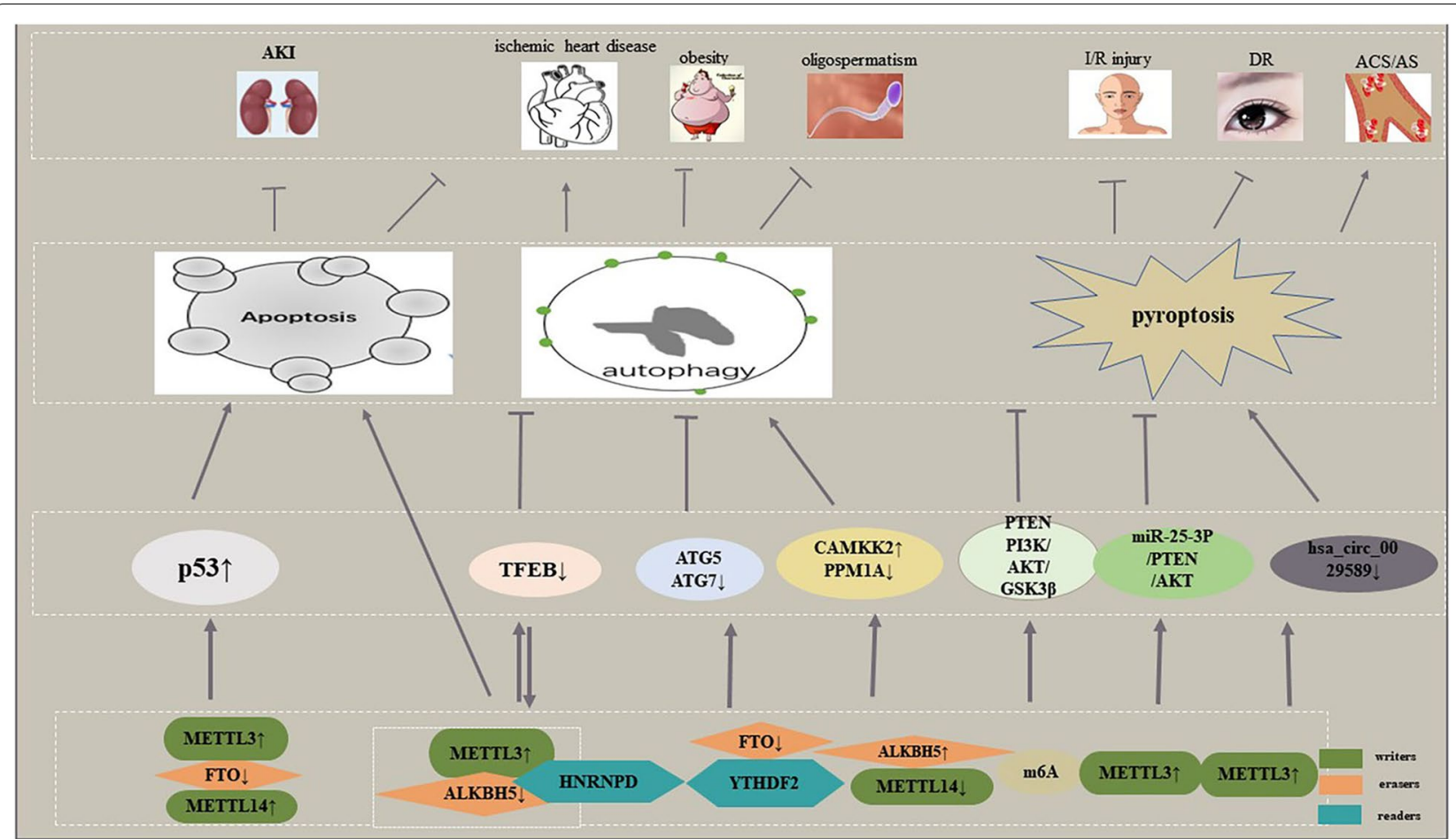

Fig. $3 \mathrm{M} 6 \mathrm{~A}$ and programmed cell death in non-cancer diseases. Enhanced m6A promotes apoptosis to inhibit the development of AKI and ischemic heart diseases. The latter can be aggravated by inhibition of TFEB-mediated autophagy, and TFEB can regulate METTL3 and ALKBH5 expression. M6A regulators suppress the progression of obesity and oligospermatisms via autophagy. M6A regulates PTEN/PI3K/AKT/GSK3 3 to inhibit pyroptosis, thus fighting against I/R injury. METTL3 upregulation modulates the development of DR and AS/ACS through pyroptosis

apoptosis rates through LNC942-METTL14-CXCR4/ CYP1B1 signaling axis [94]. Epigenetically, FTO depletion via shRNA lentiviral infection manipulates the downstream proapoptosis gene BNIP3 via a YTHDF2independent mechanism, resulting in breast cell apoptosis in vivo and in vitro [95]. Similar results observed by Zhou et al. indicated that silencing FTO enhances p53 mRNA and protein levels, aggravating excessive apoptosis after cisplatin-induced acute kidney injury (AKI) [96]. As shown by Song et al. they demonstrated that when cardiomyocytes were subjected to hypoxia/ reoxygenation, induced METTL3, but not METTL14, accounting for abnormal m6A formation, finally enhanced cell apoptosis; ALKBH5, rather than FTO, executing its demethylation tasks, ultimately repressed apoptosis [97]. Functioning as an apoptotic activator, m6A seems to be regarded as an inhibitor as well. For instance, METTL3 knockdown at the post-transcriptional level recovered the expression of ZNF750 by maintaining mRNA stability, significantly increasing the proportion of apoptotic cells dependent on FGF14 in NPC cells, and vice versa [98]. These results revealed that $\mathrm{m} 6 \mathrm{~A}$ is actively involved in cell apoptosis and probable to evaluate disease outcomes. Nevertheless, it is still possible that m6A-mediated apoptosis could have additional effects that remain to be discovered.

\section{M6A and autophagy}

Autophagy, an emerging PCD pathway, is tightly influenced by distinct stimulators and suppressors through frequently altered autophagy-related (ATG) proteins and transcription factors. In 2018, the relationship between autophagy and m6A was first described. ULK1 is a protein kinase that is motivated by autophagy. The m6A distribution within its transcripts can be abrogated by FTO, thus promoting the production of ULK1 protein, LC3BII and autophagic flux owing to a YTHDF2-regulated mechanism, which symbolizes the initiation of autophagy [99]. M6A serves as an inhibitor that enhanced METTL3 reduces autophagic flux via regulating TFEB levels and transcriptional activity, while ALKBH5 does the opposite effect in H/R-treated cardiomyocytes. Given both the contradictory role in TFEB and that it reacts on these m6A enzymes toward completely distinct directions, ALKBH5 expression is upregulated, and METTL3 is not [97]. Autophagy has been known to regulate adipose mass and adipogenesis; FTO was firstly familiar for regulating lipid metabolism. 
It is striking to confirm that FTO reduction, with YTHDF2-mediated mRNA decay and ATG5 and ATG7CEBPB signaling transmitted, obviously decreases the quantity of steady-state autophagosomes-a positive correlation between FTO and autophagy, compared to the control cells. Furthermore, attenuated fat accumulation via anti-autophagy activated by FTO deficiency is of critical functional significance for the prevention and elimination of the increasingly detrimental effects of obesity [100]. In contrast, FTO upregulation caused by low-level arsenic exposure impaired p62-mediated autophagy and autophagic degradation and formed a positive feedback loop to accumulate FTO, thus facilitating tumorigenesis in keratinocytes. This article found that IGF2BPs could reverse the effect of FTO and need further investigation [101].

With m6A engaged in the differentiation of embryonic stem cells (ESCs), sex determination, and spermatogenesis, what about those m6A modulators who are implicated in the differentiation of Leydig cells (LCs) and testosterone synthesis? The answer is that METTL14 and ALKBH5, one reduction and the other increment lead to reduced m6A levels, resulting in enhanced CAMKK2 (calcium/calmodulin-dependent protein kinase 2, beta) transcript but decreased PPM1A (protein phosphatase $1 \mathrm{~A}$, magnesium dependent, alpha isoform) protein, and intensifying the autophagy phenotype, e.g., upregulated LC3 puncta, based on (AMP-activated protein kinase) AMPK-ULK1 signals, giving assistance to the production of testosterone, irrespective of LCs age. These findings will facilitate an understanding of the importance of $\mathrm{m} 6 \mathrm{~A}$ in autophagy for the purpose of targeting azoospermatism and oligospermatism patients [102].

In parallel, autophagy also plays a role in m6A modifications. Recently, compelling evidence has proven that inhibition of FTO, targeting PD-1, CXCR4, and SOX10 in an m6A/YTHDF2-mediated manner, threatens to interfere with the malignant transformation of melanoma cells and growth of melanoma, and downregulation of the essential autophagy genes ATG5 or ATG7 reduces starvation-induced FTO and PD-1 expression, as well as NF- $\mathrm{KB}$ activity under metabolic stress, suggesting that upregulated FTO advancing melanoma tumorigenesis is induced by autophagy and the NF- $\mathrm{kB}$ pathways [103]. These findings highlight the interplay between autophagy and $\mathrm{m} 6 \mathrm{~A}$ modulators, pointing to diversified functions according to the state of the body. Despite the increasing appreciation of the biological significance regarding $\mathrm{m} 6 \mathrm{~A}$ modifications, the global effects of m6A regulators on the transcription and translation of more autophagy-related genes, combined with molecular determinants conferring RNA specificity, remain poorly understood. In turn, it may be very interesting to examine whether autophagy can regulate additional m6A components.

\section{M6A and pyroptosis}

Pyroptosis, a lytic form of cell death whose definitive hallmark genes are NLR pyrin domain containing 3 (NLRP3), apoptotic speck-like protein containing CARD (ASC), cleaved Caspase-1, Gasdermin-D (GsdmD) p30, IL-1 $\beta$ and IL-18, plays a prominent role in response to infection, ultimately fueling inflammation. Phosphatase and tensin homolog (PTEN) is regarded as a classic tumor suppressor. The latest studies have elucidated the regulation of PTEN on pyroptosis in human diseases. Notably, PTEN mRNA, which contains m6A sites, is involved in hypothermia-activated PI3K/Akt/GSK-3 $\beta$ signaling, downregulating the expression of pyroptosisrelated proteins, i.e., NLRP3 and ASC, and the secretion of proinflammatory cytokines, further protecting hippocampal neurons against $\mathrm{H} / \mathrm{R}$-induced pyroptosis upon hypoxia/reoxygenation (H/R) [104]. The participation of METTL3-mediated m6A in the pyroptosis of diabetic retinopathy (DR) was recently reported. Inhibition of METTL3, in line with miR-25-3p ablation, induced serious high glucose-fueled RPE cell apoptosis and pyroptosis and depressed cell viability by regulating the miR-25-3p/PTEN/Akt signaling cascade in a DGCR8dependent manner [105]. Beyond doubt, this discovery will bring new opportunities for the future treatment of DR through pointing at METTL3. According to Guo et al., IFN regulatory factor (IRF)-1, an IFN-inducible transcription factor, was validated to promote pyroptosis and inflammation in cultured macrophages from patients with atherosclerosis (AS) and acute coronary syndrome (ACS) by significantly decreasing the expression of hsa circ_0029589 but elevating its m6A level, along with METTL3 expression. That is to say, m6A modifications perform pivotal parts in IRF-1-induced macrophage pyroptosis [106]. Although investigations on IRF-1 in circRNA from macrophages are still in infancy, this newly discovered regulatory network is expected to provide promising therapeutic options for ACS and AS. As a consequence, these implications critically build a bridge between m6A and pyroptosis-related components, suggesting that m6A modifications on mRNAs and ncRNAs exhibit important functions in cell pyroptosis by influencing the enrichment of NLRP3, ASC, etc. Nevertheless, more research is warranted to explore other RNAs with m6A modifications in pyroptosis.

\section{M6A and ferroptosis}

Ferroptosis, a novel proinflammatory programmed cell death pathway, which plays a pivotal role in clearing malignant cells, is induced by the suppression of the 
$\mathrm{xCT} / \mathrm{GSH} / \mathrm{GPX} 4$ axis and mainly characterized by iron accretion and lipid peroxidation, accompanied by compression of mitochondrial membrane densities, reduction or disappearance of mitochondria crista and rupture of the outer mitochondrial membrane [107]. Recent studies have unraveled the relationship between m6A and ferroptotic cell death. Exosomes were released by tumor cells, and miR-4443 expression was significantly enhanced in this environment. This upregulation is associated with the chemoresistance of NSCLC to cisplatin. Further study discovered that miR-4443 mediated the production of intracellular superoxide, ROS and ferrous iron and the expression of FSP1, inhibiting ferroptosis, which was executed by decreased m6A-modified levels of FSP1 through METTL3 [108]. This finding offered a promising opportunity for increasing the efficacy of cisplatin by diminishing exosomal miR-4443 expression, enhancing FSP1 m6A deposition, and promoting ferroptotic cell death. Additionally, ferroptosis can be induced by suppression of system $\mathrm{X}_{\mathrm{c}}{ }^{-}$, a cystine/glutamate antiporter. Two major subunits of this system are SLC7A11 (solute carrier 7A11) and SLC3A2 (solute carrier 3A2). $\mathrm{Ma}$ et al. found that both of these subunits were involved in YTHDC2-regulated ferroptosis in lung adenocarcinoma (LUAD) cells. Mechanistically, YTHDC2 decreased homeobox A13 (HOXA13) mRNA stability by recognizing the m6A site in the $3^{\prime}$-UTR to suppress SLC3A2 expression, thereby inhibiting LUAD antioxidation and further limiting tumor progression. However, the detailed degradation mechanism is unclear. In summary, YTHDC2 can act as an endogenous ferroptosis motivator for LUAD patients to guide clinical therapy [109]. These researches link m6A to ferroptosis; therefore, targeting m6A to induce ferroptosis might be a promising strategy for ferroptosis-based therapy.

\section{M6A and necroptosis}

Necroptosis is a proinflammation regulated necrosis that generally results from overwhelming intra- and extracellular insults, such as TNF $\alpha$, the Fas ligand and ROS production. The morphological features are similar to nonregulated necrosis, manifesting as cytoplasmic vacuolization, organelle swelling and membrane rupture, which release DAMPs (damage-associated molecular patterns) to function. Most importantly, necroptosis can establish a cancer immunogenic microenvironment [110]. Lan et al. recently revealed that M2-polarized tumor-associated macrophages (TAMs) and METTL3 in the oxaliplatin-tolerant CRC patient tumor microenvironment (TME) are higher than those in their sensitive counterparts. Further studies revealed that infiltrated M2-TAMs increased the m6A level of TRAF5 mediated by METTL 3 and then impaired the necroptosis process, finally causing acquired oxaliplatin tolerance. This study suggests that METTL3 depletion contributes to TRAF5mediated necroptotic cell death to improve oxaliplatin resistance in CRC patients [111]. Evading apoptosis is an important means of neoplasm progression, and studies have shown that suppression of caspase8 can trigger necroptosis and that $\mathrm{m} 6 \mathrm{~A}$ regulates necroptosis, thus inducing necroptosis based on $\mathrm{m} 6 \mathrm{~A}$ regulation opens up a new avenue for anticancer strategies by blocking caspase-dependent apoptosis [112]. As mentioned earlier, the m6A modifier acts as a double-edged sword in modulating various cellular processes related to cell death, which likely hinges on abundant RNA-binding proteins (RBPs), containing m6A reader proteins and nonreader proteins, and their recognition locations. Therefore, it may be promising to identify crosstalk between m6A effectors and nonreader RBPs, or protein recruitment directly to RNAs and involvement in PCD pathways.

M6A-regulated PCD, tumorigenesis and tumor progression As mentioned above, m6A is intensively relative to the initiation and development of various cancers through PCD pathways. M6a-PCD axis acts as a double-edged sword in the pathogenesis and development of various cancers (Fig. 4).

\section{M6A-PCD Axis promoting tumorigenesis and tumor progression}

At the molecular level, YTHDF1 stimulated by hypoxiainduced HIF-1 $\alpha$ was correlated with poor survival in multiple HCC models. MeRIP-seq and proteomics analysis indicated that YTHDF1, an m6A reader, recruited initiation factors and facilitated ribosome loading to promote ATG2A and ATG14 translation through recognition of the m6A site at their CDS, thus mediating autophagy under hypoxia and $\mathrm{HCC}$ malignancy [113]. Positive alteration of Bcl-2 is driven by METTL3installed $\mathrm{m} 6 \mathrm{~A}$ in breast cancer [114]. Consistently, METTL3 overexpression, together with m6A, increased the enrichment of $\mathrm{c}-\mathrm{MYC}, \mathrm{Bcl}-2$ and PTEN proteins and undifferentiated cells, reducing apoptotic human myeloid leukemia MOLM13 cells. Pointing to myeloid tumors, these results suggest a potential therapeutic indexinhibiting METTL3 and thus inducing apoptosis [115]. Among the great number of findings, METTL3 was demonstrated to play multiple roles in gastrointestinal cancer development, such as proliferation, invasion, migration, and apoptosis [116]. More importantly, with regard to $\mathrm{m} 6 \mathrm{~A}$ and apoptosis as revealed by Wang et al. m6A depresses the expression of Bax and active caspase- 3 but promotes the synthesis of Bcl-2, thereby countering cancer cell apoptotic pathways. Compared with normal cells, METTL3 increased in gastric cancer cells [117]. The 


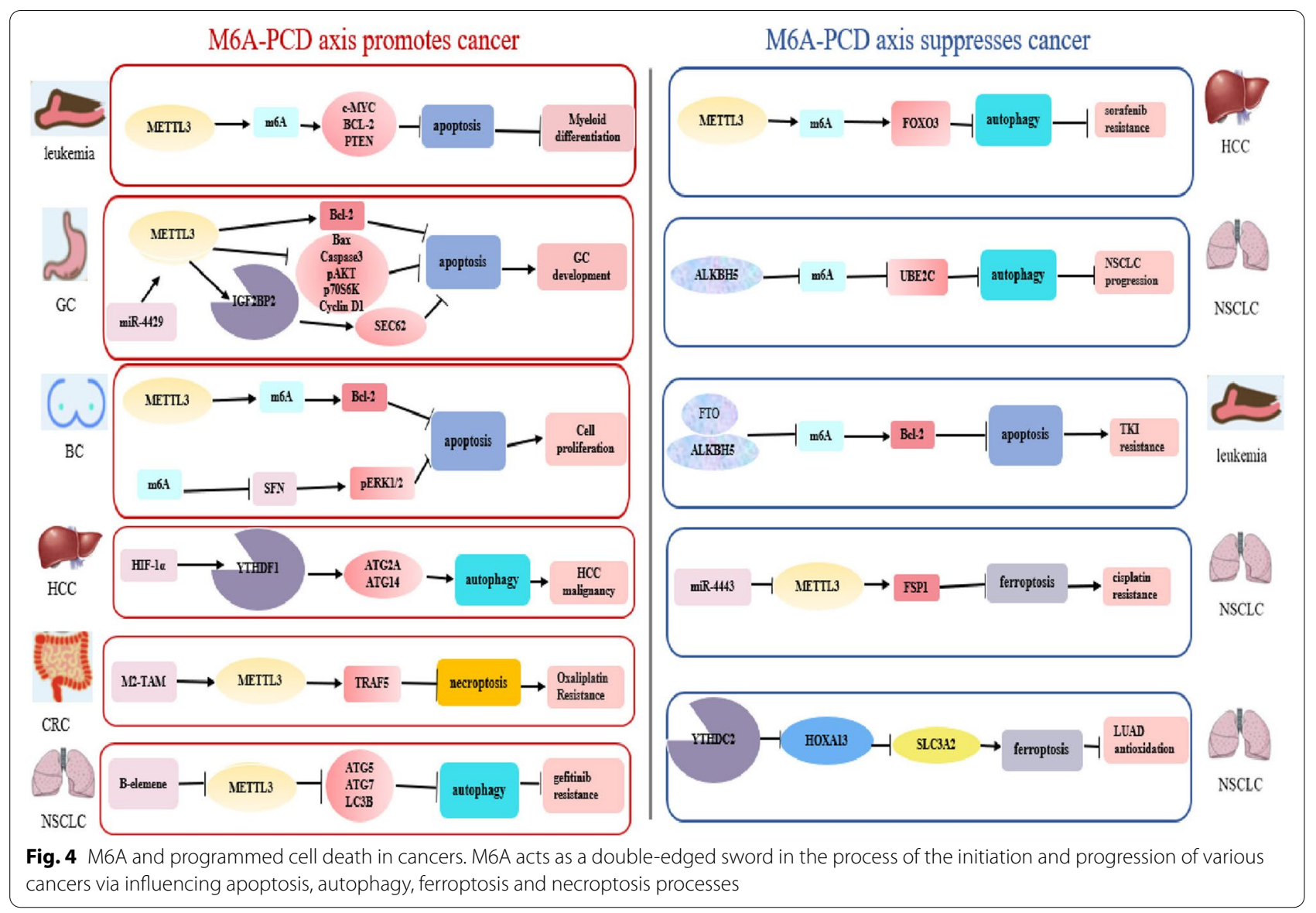

latest findings have confirmed that METTL3 hampered colorectal cancer cell necroptosis to promote oxaliplatin resistance [111]. Furthermore, m6A has an impact on autophagy through regulating autophagy-associated proteins, altering the sensitivity of cancer cells to anticancer drugs [118]. Conspicuously, METTL3 will be realized as a hallmark for cancer initiation and propagation and deserve appreciation.

Sulforaphane (SFN), a dietary phytochemical, could induce apoptosis, nitro-oxidative stress, and genotoxicity and decrease the AKT pathway in breast cancer cells. The stimulation of energy stress by SFN is determined by autophagy activation at both cytostatic and cytotoxic concentrations. Notably, SFN-mediated cell cycle arrest and cellular senescence in cancer cells might be related to suppressed m6A RNA methylation through enhancing the instability of target genes to some extent. Regarding the interaction between SFN-regulated autophagy and apoptosis, previous studies have presented comparatively distinct results: diminished autophagy facilitated apoptotic cell death in prostate, colon and breast cancer cells but functioned independently in SFN-treated pancreatic carcinoma cells [119]. Therefore, exploration of the basal association of autophagy with apoptosis regulated by $\mathrm{m} 6 \mathrm{~A}$ will assist in the identification of pathogenic mechanisms for carcinogenesis and development. Moreover, $\mathrm{m} 6 \mathrm{~A}$ is regarded as a promising candidate for cancer therapy since the initiation of malignancies is characterized by an aberrant epigenetic landscape.

\section{M6A-PCD Axis inhibiting tumorigenesis and tumor progression}

The involvement of extraordinary autophagy-related transcription factors (TFs) in m6A-governed PCD pathways has been reported. Exposed to hypoxia, FOXO3 is such one that downregulated METTL3 raised resistance to sorafenib in HCC by decreasing its mRNA stabilization in a YTHDF1-reliant fashion in that the concomitant m6A decrease enhanced the level of autophagosomes and LC3 accumulation [120]. In leukemia, some studies displayed a negative correlation between m6A and apoptosis-Bcl-2 overexpression evoked by FTO and ALKBH5 upregulation fights against apoptosis, resulting in resistance of leukemia cells to tyrosine kinase inhibitors (TKIs) [114]. METTL3 and YTHDC2 are both ferroptosis inducers that the former could raise cisplatin efficacy in 
NSCLC and the latter could inhibit lung adenocarcinoma tumorigenesis $[108,109]$.

Intriguingly, UBE2C, mediated by ALKBH5, links apoptosis (type I PCD) with autophagy (type II PCD), which coregulates autophagic apoptosis in cancer cells. Increased UBE2C expression elevated autophagic phenotypes and drastically governed cancer cell invasive growth, EMT panels and apoptotic cell death in NSCLC, revealing the roles of motivation of autophagy genes in autophagic and apoptotic functions [121].

In accordance with these findings, the interplay between $\mathrm{m} 6 \mathrm{~A}$ and PCD in the process of tumorigenesis and progression requires attentions, and its investigation will not only contribute to understanding the cancer epigenome, including the m6A signaling network, but also to identifying anticancer targets. More researches on m6A and other PCD signaling pathways in various cancers are urgently needed in the future.

\section{Clinical application of the target-based m6A-PCD Axis}

Emerging data have indicated that $\mathrm{m} 6 \mathrm{~A}$ modifiers are generally dysregulated in various diseases, characterized by aberrant global m6A abundance, gene expression levels of m6A writers, erasers and readers, and mutations in $\mathrm{m} 6 \mathrm{~A}$ sites, as well as epitranscriptome changes upon external cues, thereby providing promising biomarkers for clinical diagnosis, treatment and prognosis [122]. Given the multiple functions of m6A-regulated proteins in PCD, making elaboration on how they interact in pathological processes will be propitious to discover specific potential biomarkers and therapeutic targets for cancer diagnosis, treatment and prognostic evaluation, as well as drug resistance (Table 1). In particular, by focusing on the links between m6A and cell apoptosis, autophagy, ferroptosis and necroptosis in cancer, we cannot ignore the potential value of $\mathrm{m} 6 \mathrm{~A}$ in antitumor actions. In summary, the m6A-mediated PCD axis has become a vital mechanism during pathological processes. As RNAs contain multiple m6A sites in PCD pathways, targeting this interplay shows more diagnostic and therapeutic advantage and potential than targeting a single m6A. Concurrently, by expanding our view of $\mathrm{m} 6 \mathrm{~A}$ and $\mathrm{m} 6 \mathrm{~A}$-regulated PCD, we will broaden our understanding of a variety of m6A protein molecular functions and their application as prognostic indicators of clinical severity.

\section{Diagnostic potential}

As performed by RNA-seq assay in pancreatic cancer (PC), ALKBH5 overexpression and thus PER1 demethylation altered related apoptotic transcriptome in a YTHDF2-dependent way, which suppressed tumoral growth. Therefore, ALKBH5 and PER1 may be biomarkers for PC diagnosis and therapy [134]. LNC942 acted as a tumor-driver gene in breast cancer cells. Specifically, elevated METTL14 recruited by LNC 942 promoted cell proliferation and inhibited cell apoptosis through its target genes like CXCR4 and CYP1B1. It is noteworthy that the METTL14-apoptosis axis plays role in early diagnosis, treatment and prognosis evaluation for breast cancer [94]. As previous work reported, knock-down of YTHDF2 and METTL3 significantly triggered cell apoptosis by mediating LHPP and NKX3-1 mRNA levels, and this finding may contribute to the expansion of potential diagnosis or therapeutic markers of prostate cancer [125]. Additionally, circRAB11FIP1 overexpression augmented m6A demethylation of ATG5 and ATG7 to promote autophagy through sponging FTO to aggravate malignant behavior of ovarian cancer [135].

\section{Therapeutic potential}

METTL3 is frequently associated with many types of cancers, such as gastric cancer [12], bladder cancer [9], and colorectal cancer [141]. Accordingly, He et al. found that upregulated METTL3 promoted the stability of SEC62 mRNA via IGF2BP1 in GC cell lines. Moreover, miR-4429 might inhibit the expression of SEC62 by controlling METTL3. Furthermore, miR-4429 or silencing SEC62 blocked tumor cell proliferation and facilitated apoptosis, suggesting that miR-4429 and its downstream gene METTL3 are potential targets for gastric cancer prevention and management [124]. In addition, mutant $\mathrm{m} 6 \mathrm{~A}$ is related to leukemogenesis by governing PCD pathways. In Paris et al.s AML study, elevated YTHDF2 was found to play a pivotal role in leukemia initiation and propagation. At the molecular level, YTHDF2 helps the functional integrity of leukemic stem cells (LSCs) by reducing the half-life of corresponding m6A targets containing the tumor necrosis factor receptor Tnfrsf2, whose elevation stimulates cell apoptosis in Ythdf2-deficient LSCs. In particular, YTHDF2 deletion contributes to HSC activity, while YTHDF2 does not derail normal hematopoiesis. In accordance, YTHDF2 loss will be a critical target for treating AML through bringing about HSC expansion and promoting myeloid reconstitution, specifically, selectively eradicating malignant LSCs [132]. The demethylase ALKBH5 was downregulated in osteosarcoma, and m6A methylation of pre-miR-181b-1 and YAP-mRNA was upregulated. This promoted osteosarcoma cell growth, migration, and invasion and suppressed cell apoptosis. In contrast, ALKBH5 overexpression had antitumor effects. Therefore, ALKBH5-based m6A demethylation opens up a new strategy for replacement therapy against osteosarcoma through m6A-correlated posttranscriptional regulation of pre-miRNA-181b-1 and Yes-associated protein 1 (YAP) [133]. R-2-Hydroxyglutarate (R-2HG), usually 
Table 1 The clinical application of m6A and PCD

\begin{tabular}{|c|c|c|c|c|c|}
\hline $\begin{array}{l}\text { M6A associated } \\
\text { molecules }\end{array}$ & $\begin{array}{l}\text { Types of } \\
\text { programmed cell } \\
\text { death pathways }\end{array}$ & Molecular mechanisms & $\begin{array}{l}\text { The corresponding } \\
\text { cancers }\end{array}$ & Clinical application & Ref. \\
\hline FTO & Apoptosis & Increased MZF1 expression & LUSC & Prognosis and therapy & {$[123]$} \\
\hline METTL3 and IGFBP2 & Apoptosis & $\begin{array}{l}\text { Promoted the stability of } \\
\text { SEC62 mRNA }\end{array}$ & Gastric cancer & $\begin{array}{l}\text { Prevention and manage- } \\
\text { ment }\end{array}$ & [124] \\
\hline METTL3 and YTHDF2 & Apoptosis & $\begin{array}{l}\text { Mediated LHPP and } \\
\text { NKX3-1 mRNA }\end{array}$ & Prostate cancer & Diagnosis and therapy & {$[125]$} \\
\hline HNRNPC & Apoptosis & $\begin{array}{l}\text { Downregulated miR-21 } \\
\text { and AKT phosphorylation }\end{array}$ & Ovarian cancer & Prognosis & {$[126]$} \\
\hline METTL3 & Autophagy & $\begin{array}{l}\text { Stimulated autophagy of } \\
\text { ARHGAP5-AS1 or destabi- } \\
\text { lizing ARHGAP5 }\end{array}$ & Gastric cancer & $\begin{array}{l}\text { Anti-chemoresistance and } \\
\text { prognosis }\end{array}$ & {$[127]$} \\
\hline METTL3 & Apoptosis & $\begin{array}{l}\text { Impinged on critical cel- } \\
\text { lular processes }\end{array}$ & Cervical cancer & Radio/ chemo-resistance & {$[128,129]$} \\
\hline METTL14 & Apoptosis & $\begin{array}{l}\text { Targeted CXCR4 and } \\
\text { CYP1B1 }\end{array}$ & Breast cancer & $\begin{array}{l}\text { Diagnosis, therapy and } \\
\text { prognosis }\end{array}$ & [94] \\
\hline WTAP & Apoptosis & $\begin{array}{l}\text { Affected PI3K/AKT signal- } \\
\text { ing }\end{array}$ & AML & Prognosis & [130] \\
\hline WTAP & Apoptosis & Regulated DUSP6 & NKTCL & Drug resistance & [131] \\
\hline YTHDF2 & Apoptosis & $\begin{array}{l}\text { Reduced the half-life of } \\
\text { Tnfrsf2 }\end{array}$ & $\mathrm{AML}$ & Therapy & [132] \\
\hline ALKBH5 & Apoptosis & $\begin{array}{l}\text { Reduced m6A-methylated } \\
\text { pre-miR-181b-1 and YAP }\end{array}$ & Osteosarcoma & Therapy & [133] \\
\hline $\begin{array}{r}\text { ALKBH5 } \\
\text { And YTHDF2 }\end{array}$ & Apoptosis & & Pancreatic cancer & Diagnosis and therapy & [134] \\
\hline FTO & Autophagy & $\begin{array}{l}\text { Augmented m6A demeth- } \\
\text { ylation of ATG5 and ATG7 }\end{array}$ & Ovarian cancer & Diagnosis and therapy & [135] \\
\hline FTO & Apoptosis & $\begin{array}{l}\text { Caused inhibition of MYC/ } \\
\text { CEBPA signaling }\end{array}$ & Glioma & Anti-cancer activity & [136] \\
\hline FTO & Apoptosis & $\begin{array}{l}\text { Reduced hypomethylation } \\
\text { and Bcl-2 transcripts }\end{array}$ & AML & Drug resistance & [137] \\
\hline M6A & Apoptosis & $\begin{array}{l}\text { Regulated the Daple/ } \beta \text { - } \\
\text { catenin/ABCC9 signaling } \\
\text { pathway }\end{array}$ & NPC & Drug resistance & [138] \\
\hline RBM15 & Apoptosis & $\begin{array}{l}\text { Upregulated TMBIM6 m6A } \\
\text { modification }\end{array}$ & LSCC & Therapy and Prognosis & [139] \\
\hline FTO & Autophagy & $\begin{array}{l}\text { Reduced m6A methylation } \\
\text { of the critical protu- } \\
\text { morigenic melanoma cell- } \\
\text { intrinsic genes containing } \\
\text { PD-1(PDCD-1), CXCR4 and } \\
\text { SOX10 and maintaining } \\
\text { their mRNA stability }\end{array}$ & Melanoma & $\begin{array}{l}\text { Immune therapy resist- } \\
\text { ance }\end{array}$ & [103] \\
\hline M6A writers & Apoptosis & $\begin{array}{l}\text { Correlated with drugs } \\
\text { which targeted oncogenic } \\
\text { related pathways, such as } \\
\text { MAPK, EGFR, and mTOR } \\
\text { signaling pathways }\end{array}$ & Colorectal cancer & $\begin{array}{l}\text { Immune therapy resist- } \\
\text { ance }\end{array}$ & [140] \\
\hline
\end{tabular}

LUSC lung squamous cell carcinoma, NKTCL Natural killer/T-cell lymphoma, DUSP6 dual-specificity phosphatases 6, YAPYes-associated protein 1

an oncogenic metabolite, exhibits anticancer activity in leukemia and glioma patients with IDH1/2 mutations. In terms of mechanism, R-2HG elevated global m6A by inhibiting FTO in R-2HG-sensitive cells, causing inhibition of MYC/CEBPA signaling and thus resulting in cancer cell cycle arrest and apoptosis. S-2HG showed the same effect. The inhibition of FTO enzymatic activity might enhance the response to chemotherapeutic agents. Overall, this work provided previously unrecognized insights into 2HG-type molecules or selective FTO suppressors, alone and specifically combined with other agents, for example, hypomethylating AZA, which 
holds therapeutic potential in IDH wild-type tumors with high FTO [136].

\section{Prognostic potential}

As elucidated by Liu et al. abnormal m6A motifs in lung squamous cell carcinoma (LUSC) were attributed to the FTO gene, which reduced its m6A levels and mRNA stability, contributing to increased MZF1 expression and thus oncogenic impacts. Furthermore, FTO deletion significantly promoted the apoptosis and repressed the proliferation and invasion of L78 and NCI-H520 cells. This finding supported the role of FTO as a potential prognostic factor and therapeutic target [123]. Another study on miRNAs showed the relevance between m6A and apoptosis in ovarian cancer cells. Overexpression of miR-744-5p reduced the enrichment of nuclear factor I X (NFIX) and HNRNPC by modulating their mRNA and protein expression. The former caused decreased $\mathrm{Bcl} 2$ levels, exerting proapoptotic effects on cancer cells, whereas the latter downregulated miR-21 and AKT phosphorylation. More importantly, high miR-744 prolonged the median disease-free survival of ovarian serous cystadenocarcinoma patients, indicating the significance of HNRNPC as a prognostic target [126].

Furthermore, overexpression of m6A writer WTAP modulated the AML cell cycle, behaving as G1/S-phase arrest, further inhibiting the cell proliferation and colony formation. Bioinformatics analysis revealed that WTAP knockdown significantly affected PI3K/AKT signaling. On the other hand, WTAP acts as a predictor for poor prognosis in AML. A previous study conducted by Bansal et al. found that deficiency of WTAP upregulated the apoptosis ratio of K562 and HL-60 cells after etoposide challenge [130]. In summary, the prognostic and epigenetic roles of $\mathrm{m} 6 \mathrm{~A}$ regulators in AML have been extensively investigated. The present studies mainly focus on m6A and AML cell apoptosis, and the interaction between $\mathrm{m} 6 \mathrm{~A}$ and other PCDs and whether m6A exerts an effect on additional hematopoietic malignances might be an intriguing area of research. Moreover, Wang at el. first demonstrated that the m6A writer RBM15 was overexpressed in laryngeal squamous cell carcinoma (LSCC) and facilitated the progression of LSCC by regulating m6A-based TMBIM6 mRNA, and TMBIM6 m6A modification regulated cell apoptosis. The downregulation of IGF2BP3 inhibited TMBIM6 expression by decreasing its stability, moreover, high RBM15 levels were associated with poor prognosis of LSCC. Taken together, the RBM15/IGF2BP3/TMBIM6 axis could be promising therapeutic and prognostic targets for LSCC patients [139].
Collectively, these results provide us with a research insight: m6A regulators modulate programmed cell death, especially cell apoptosis in diverse cancers. The limited knowledge on the m6A molecule paradigm should inspire researchers to conduct numerous in-depth studies of the role of m6A modifications and PCD in various diseases, which will generate new therapeutic modalities. Therefore, they themselves and their upstream/ downstream regulators might act as diagnostic, therapeutic and prognostic biomarkers.

\section{Therapeutic resistance}

Resistance to therapy strategies (e.g., immunotherapy, radiotherapy and chemotherapy) severely hinder the treatment of cancer patients. Notably, m6A might be considered a potential determinant to cope with therapy resistance through multiple pathways, one of which is the control of downstream adaptive responses (autophagy, apoptosis and the like) [114].

\section{Chemotherapy resistance}

In gastric cancer cells resistant to chemotherapy, ARHGAP5-AS1 was recognized as an elevated lncRNA, and knocking it down improved chemoresistance. Interestingly, its upregulation was resulted from autophagy inhibition, caused ARHGAP5 transcript activation in the nucleus. Additionally, METTL3 was recruited to motivate the formation of m6A-methylated ARHGAP5 mRNA in the cytoplasm, implying that autophagic degradation of ARHGAP5-AS1 and inhibition of ARHGAP5 could be expected to reverse chemoresistance. As expected, this result produced a promising strategy to improve chemosensitivity and prognosis in gastric cancer by stimulating autophagy of ARHGAP5-AS1 or destabilizing ARHGAP5 caused by METTL3 removal [127]. WTAP, another MTC component, protects natural killer/ T-cell lymphoma (NKTCL) cells from Bax-modulated apoptosis induced by cisplatin (DDP) via regulation of dual-specificity phosphatases 6 (DUSP6). That is, ablation of WTAP augmented the chemosensitivity of NKTCL by decreasing the drug resistance-associated proteins MRP1 and P-gp [131]. Saikosaponin-d (SsD), an FTO inhibitor, improved the efficacy of nilotinib and PKC412 by reducing FTO-mediated $\mathrm{m}^{6} \mathrm{~A}$ hypomethylation and $\mathrm{Bcl}-2$ transcripts in AML-resistant patients [137]. High m6A, accompanied with TRIM11 overexpression in NPC drugresistant cells, inhibited apoptosis in vitro and positively regulated the Daple/ $\beta$-catenin/ABCC9 signaling pathway to impede cisplatin sensitivity. Therefore, reducing m6A levels and further TRIM11 stability will improve chemoresistant NPC therapy [138]. 


\section{Radiotherapy resistance}

Radiotherapy is another important treatment method for patients with advanced cancers; however, radioresistance regularly gives rise to treatment failure. Several researches have shown that METTL3 impinges on critical cellular processes including apoptosis to result in resistance to chemo- and radiotherapy in pancreatic cancer cells. Moreover, m6A modifications on lncRNA MALAT1 could be associated with radioresistance/ chemoresistance by impairing the apoptosis response in cervical cancer $[128,129]$.

\section{Immunotherapy resistance}

Immune checkpoint blockade (ICB) therapy is a historic revolution in cancer treatment, but only a small percentage of patients respond to it. The mechanism which m6A regulates immunotherapy resistance is under scrutiny. M6A is one of four major RNA adenosine modifications, and their functions are regulated by writers. RNA modification writers were divided into two expression patterns detected by WM_Score: The WM_Score-high group exerted effects on worse patient overall survival and recruitment of inhibitory immune cells and the WM_Score-low group showed effects on a survival advantage, apoptosis, and cell cycle signaling reducing targeted drug resistance. It is worth noting that analysis of the WM_Score could increase the efficacy of PD-L1 blockade by targeting associated writers [140]. The demethylases, FTO and ALKBH5 reduce the response to antiPD-1 blockade and accelerate melanoma tumorigenesis by reducing $\mathrm{m} 6 \mathrm{~A}$ methylation of critical protumorigenic melanoma cell-intrinsic genes [103, 142].

Treatment failure arising from primary or acquired resistance is a major hurdle for the survival of cancer patients, particularly advanced patients. Thus, clarifying the molecular mechanisms of therapy resistance in various malignancies is significant for exploring personalized and precise therapeutic approaches. As mentioned above, these findings insinuated that targeting m6A molecules might be an opportunity for resolving therapy resistance.

\section{Conclusion}

M6A is assembled by m6A methyltransferases, recognized by reader proteins, and abolished by $\mathrm{m} 6 \mathrm{~A}$ demethylases. It remains possible that novel m6A modifiers still need to be identified [143]. Interconnection of apoptosis and ferroptosis, apoptosis and autophagy, and autophagy as well as ferroptosis indicated that m6A could fine-tune more programmed cell death pathways [144, 145]. Programmed cell death by m6A on mRNA and ncRNAs regulates the oncogenesis and development of cancers. However, whether and how
m6A proteins and m6A impact $\mathrm{PCD}$ remain controversial. For instance, m6A exhibits paradoxical rolesMETTL3 serves as an apoptotic driver in HG (high glucose)-reliant human lens epithelial cells (HLECs), while WTAP acts as a suppressor in YTS and SNK-6 cells, two human NKTCL cell lines [146, 147]. There are two main reasons for this discrepancy. One possible explanation is that m6A mRNA status and function are correlated with varied physiological contexts, and the other is the effects of its up/downstream. M6A-associated targets would provide a new direction for clinical diagnosis, treatment, prognosis, and therapy resistance in cancers.

\section{Abbreviations}

ACS: Acute coronary syndrome; AKI: Acute kidney injury; AMPK: AMP-activated protein kinase; AS: Atherosclerosis; ASC: Apoptotic speck-like protein containing CARD; CAMKK2: Calcium/calmodulin-dependent protein kinase 2; CDS: Coding sequences; DAMPs: Damage-associated molecular patterns; DDP: Cisplatin; DR: Diabetic retinopathy; DUSP6: Dual-specificity phosphatases 6; elF3: Eukaryotic translation initiation factor 3; ESCs: Embryonic stem cells; FTO: Fat mass and obesity-associated protein; GC: Gastric cancer; GsdmD: Gasdermin-D; HCC: Hepatocellular carcinoma; HG: High glucose; HLECs: Human lens epithelial cells; HNRNPA2/B1: Heterogeneous nuclear ribonucleoprotein A2/B1; HOXA13: Homeobox A13; H/R: Hypoxia/reoxygenation; ICB: Immune checkpoint blockade; IGF2BP1/2/3: Insulin-like growth factor 2 mRNA-binding proteins; IRF: IFN regulatory factor; LCs: Leydig cells; LSCC: Laryngeal squamous cell carcinoma; LSCs: Leukemic stem cells; LUAD: Lung adenocarcinoma; LUSC: Lung squamous cell carcinoma; MALDI-TOF-MS: Matrix-assisted laser desorption/ionization time of flight mass spectrometry; METTL3: Methyltransferase-like protein 3; MTC: Methyltransferase complex; ncRNAs: Noncoding RNAs; NFIX: Nuclear factor I X; NKTCL: Natural killer/T-cell lymphoma; NLRP3: NLR pyrin domain containing 3; NSUn2: NOP2/Sun domain family; PA-m6A-seq: Photo-cross-linking-assisted m6A sequencing; PCD: Programmed cell death; PPM1A: Protein phosphatase 1A; PTEN: Phosphatase and tensin homolog; RBM15/15B: RNA binding motif protein 15/15B; RBPs: RNAbinding proteins; R-2HG: R-2-Hydroxyglutarate; R-M: Restriction-modification; SAM: S-adenosyl methionine; SFN: Sulforaphane; SLC3A2: Solute carrier 3A2; SLC7A11: Solute carrier 7A11; SsD: Saikosaponin-d;TAMs: Tumor-associated macrophages; TFs: Transcription factors; TICs: Tumor-initiating cells; TKIs: Tyrosine kinase inhibitors; TME: Tumor microenvironment; UBE2C: Ubiquitin-conjugating enzyme E2C; VIRMA: Vir like m6A methyltransferase associated; WTAP: Wilms tumor 1-associating protein; YAP: Yes-associated protein 1; YTHDC1:YTH domain containing 1; YTHDF1: YTH domain family protein 1; ZC3H13: Zinc finger CCCH-type containing 13.

\section{Authors' contributions}

$\mathrm{LL}$ wrote the manuscript, and $\mathrm{HL}$ summarized the table and prepared figures. $J \mathrm{~L}$ reviewed the manuscript and revised the manuscript. DH, YW, WS, JZ reviewed the manuscript and prepared figures. SY revised the manuscript. JZ conceived the projects and revised the manuscript. All authors reviewed the manuscript and approved the final manuscript.

\section{Funding}

This work was funded by the National Natural Science Foundation of China (No. 82170138, 81870105, 81920108004, 81770107 and 82003286), National Key Research and Development Program of China (2018YFA0107800), Natural Science Foundation of Hunan Province (No. 2021JJ30022, 2019JJ20014 and 2021 JJ40054), Scientific Research Fund Project of Hunan Provincial Health Commission (No. 20201921) and the Changsha Municipal Natural Science Foundation (No. kq20140421).

Availability of data and materials

Not applicable, all information in this review can be found in the reference list. 


\section{Declaratons}

\section{Ethics approval and consent to participate}

No ethics approval was required for this review that did not involve patients or patient data.

\section{Consent for publication}

All authors consent to publication.

\section{Competing interests}

The authors have no competing interests to declare.

\section{Author details}

'Department of Clinical Laboratory, Shenzhen Traditional Chinese Medicine Hospital, Shenzhen 518033, Guangdong, China. ${ }^{2}$ The First Affiliated Hospital, Department of Rheumatology, Hengyang Medical School, University of South China, Hengyang 421001, Hunan, China. ${ }^{3}$ Hunan Province Key Laboratory of Basic and Applied Hematology, Molecular Biology Research Center \& Center for Medical Genetics, School of Life Sciences, Central South University, Changsha 410078, Hunan, China. ${ }^{4}$ Department of Dermatology, Hunan Key Laboratory of Skin Cancer and Psoriasis, Hunan Engineering Research Center of Skin Health and Disease, Xiangya Clinical Research Center for Cancer Immunotherapy, Xiangya Hospital, Central South University, Changsha 410008, Hunan, China. ${ }^{5}$ The First Affiliated Hospital, Department of Hematology, Hengyang Medical School, University of South Chinal, Hengyang 421001, Hunan, China.

Received: 29 August 2021 Accepted: 12 January 2022

Published online: 28 January 2022

\section{References}

1. Yang J, Chen J, Fei X, Wang X, Wang K. N6-methyladenine RNA modification and cancer. Oncol Lett. 2020;20(2):1504-12.

2. Jiang H, Cao K, Fan C, Cui X, Ma Y, Liu J. Transcriptome-wide highthroughput m6A sequencing of differential m6A methylation patterns in the human rheumatoid arthritis fibroblast-like synoviocytes cell line MH7A. J Inflamm Res. 2021;14:575-86.

3. Wang T, Kong S, Tao M, Ju S. The potential role of RNA N6-methyladenosine in cancer progression. Mol Cancer. 2020;19(1):88.

4. Chen $X Y$, Zhang J, Zhu JS. The role of $m(6) A$ RNA methylation in human cancer. Mol Cancer. 2019;18(1):103.

5. He L, Li H, Wu A, Peng Y, Shu G, Yin G. Functions of N6-methyladenosine and its role in cancer. Mol Cancer. 2019:18(1):176.

6. Xi L, Carroll T, Matos I, Luo JD, Polak L, Pasolli HA, et al. m6A RNA methylation impacts fate choices during skin morphogenesis. Elife. 2020;9:e56980.

7. Wang J, Wang J, Gu Q, Ma Y, Yang Y, Zhu J, et al. The biological function of m6A demethylase ALKBH5 and its role in human disease. Cancer Cell Int. 2020:20:347.

8. Anita R, Paramasivam A, Priyadharsini JV, ChitraS.The m6A readers YTHDF1 and YTHDF3 aberrations associated with metastasis and predict poor prognosis in breast cancer patients. Am J Cancer Res. 2020;10(8):2546-54.

9. Han J, Wang JZ, Yang X, Yu H, Zhou R, Lu HC, et al. METTL3 promote tumor proliferation of bladder cancer by accelerating pri-miR221/222 maturation in m6A-dependent manner. Mol Cancer. 2019;18(1):110.

10. Cheng $M$, Sheng $L$, Gao $Q$, Xiong $Q$, Zhang $H$, Wu M, et al. The m(6)A methyltransferase METTL3 promotes bladder cancer progression via AFF4/NF-kB/MYC signaling network. Oncogene. 2019;38(19):3667-80.

11. Chen Y, Peng C, Chen J, Chen D, Yang B, He B, et al. WTAP facilitates progression of hepatocellular carcinoma via m6A-HuR-dependent epigenetic silencing of ETS1. Mol Cancer. 2019;18(1):127.

12. Wang Q, Chen C, Ding Q, Zhao Y, Wang Z, Chen J, et al. METTL3mediated m(6)A modification of HDGF mRNA promotes gastric cancer progression and has prognostic significance. Gut. 2020;69(7):1193-205.

13. Gu C, Wang Z, Zhou N, Li G, Kou Y, Luo Y, et al. Mettl14 inhibits bladder TIC self-renewal and bladder tumorigenesis through N(6)-methyladenosine of Notch1. Mol Cancer. 2019;18(1):168.

14. Qing Y, Dong L, Gao L, Li C, Li Y, Han L, et al. R-2-hydroxyglutarate attenuates aerobic glycolysis in leukemia by targeting the $\mathrm{FTO} / \mathrm{m}(6) \mathrm{A}$ PFKP/LDHB axis. Mol Cell. 2021;81(5):922-39.e9.
15. Wang JY, Chen LJ, Qiang P. The potential role of N6-Methyladenosine (m6A) demethylase fat mass and obesity-associated gene (FTO) in human cancers. Onco Targets Ther. 2020;13:12845-56.

16. Liu T, Wei Q, Jin J, Luo Q, Liu Y, Yang Y, et al. The m6A reader YTHDF1 promotes ovarian cancer progression via augmenting EIF3C translation. Nucleic Acids Res. 2020;48(7):3816-31.

17. Ni W, Yao S, Zhou Y, Liu Y, Huang P, Zhou A, et al. Long noncoding RNA GAS5 inhibits progression of colorectal cancer by interacting with and triggering YAP phosphorylation and degradation and is negatively regulated by the m(6)A reader YTHDF3. Mol Cancer. 2019;18(1):143.

18. Wang H, Xu B, Shi J. N6-methyladenosine METTL3 promotes the breast cancer progression via targeting Bcl-2. Gene. 2020;722:144076.

19. Jin D, Guo J, Wu Y, Du J, Yang L, Wang X, et al. m(6)A mRNA methylation initiated by METTL3 directly promotes YAP translation and increases YAP activity by regulating the MALAT1-miR-1914-3p-YAP axis to induce NSCLC drug resistance and metastasis. J Hematol Oncol. 2021;14(1):32.

20. Guo J, Ren W, Li A, Ding Y, Guo W, Su D, et al. Fat mass and obesity-associated gene enhances oxidative stress and lipogenesis in nonalcoholic fatty liver disease. Dig Dis Sci. 2013;58(4):1004-9.

21. Mizuno TM. Fat mass and obesity associated (FTO) gene and Hepatic glucose and lipid metabolism. Nutrients. 2018;10(11):1600.

22. De Jesus DF, Zhang Z, Kahraman S, Brown NK, Chen M, Hu J, et al. m(6) A mRNA methylation regulates human $\beta$-cell biology in physiological states and in type 2 diabetes. Nat Metab. 2019;1(8):765-74.

23. Myoung J, Ganem D. Infection of lymphoblastoid cell lines by Kaposi's sarcoma-associated herpesvirus: critical role of cell-associated virus. J Virol. 2011:85(19):9767-77.

24. Canaani D, Kahana C, Lavi S, Groner Y. Identification and mapping of N6-methyladenosine containing sequences in simian virus 40 RNA. Nucleic Acids Res. 1979;6(8):2879-99.

25. Tsai K, Courtney DG, Cullen BR. Addition of m6A to SV40 late mRNAs enhances viral structural gene expression and replication. PLoS Pathog. 2018;14(2):e1006919.

26. $\mathrm{Vu}$ LP, Cheng Y, Kharas MG. The biology of m(6)A RNA methylation in Normal and malignant hematopoiesis. Cancer Discov. 2019:9(1):25-33.

27. Yang F, He Y, Zhai Z, Sun E. Programmed cell death pathways in the pathogenesis of systemic lupus erythematosus. J Immunol Res. 2019:2019:3638562

28. Frank D, Vince JE. Pyroptosis versus necroptosis: similarities, differences, and crosstalk. Cell Death Differ. 2019;26(1):99-114.

29. Bonam SR, Wang F, Muller S. Autophagy: a new concept in autoimmunity regulation and a novel therapeutic option. J Autoimmun. 2018:94:16-32.

30. Kajarabille N, Latunde-Dada GO. Programmed cell-death by Ferroptosis: antioxidants as mitigators. Int J Mol Sci. 2019;20(19):4968.

31. Tower J. Programmed cell death in aging. Ageing Res Rev. 2015;23(Pt A):90-100.

32. Bedoui S, Herold MJ, Strasser A. Emerging connectivity of programmed cell death pathways and its physiological implications. Nat Rev Mol Cell Biol. 2020:21(11):678-95.

33. Chen KW, Demarco B, Broz P. Beyond inflammasomes: emerging function of gasdermins during apoptosis and NETosis. EMBO J. 2020;39(2):e103397.

34. Fuchs $Y$, Steller H. Programmed cell death in animal development and disease. Cell. 2011:147(4):742-58.

35. Gautheron J, Gores GJ, Rodrigues CMP. Lytic cell death in metabolic liver disease. J Hepatol. 2020;73(2):394-408.

36. Zhang C, Zhang M, Ge S, Huang W, Lin X, Gao J, et al. Reduced m6A modification predicts malignant phenotypes and augmented Wnt/ PI3K-Akt signaling in gastric cancer. Cancer Med. 2019;8(10):4766-81.

37. Fu Y, Luo GZ, Chen K, Deng X, Yu M, Han D, et al. N6-methyldeoxyadenosine marks active transcription start sites in Chlamydomonas. Cell. 2015;161(4):879-92

38. Greer EL, Blanco MA, Gu L, Sendinc E, Liu J, Aristizábal-Corrales D, et al. DNA methylation on N6-adenine in C. elegans. Cell. 2015;161(4):868-78

39. Zhang G, Huang H, Liu D, Cheng Y, Liu X, Zhang W, et al. N6-methyladenine DNA modification in Drosophila. Cell. 2015;161(4):893-906.

40. Iyer LM, Zhang D, Aravind L. Adenine methylation in eukaryotes: apprehending the complex evolutionary history and functional potential of an epigenetic modification. Bioessays. 2016;38(1):27-40.

41. Dominissini D, Moshitch-Moshkovitz S, Schwartz S, Salmon-Divon M, Ungar L, Osenberg $\mathrm{S}$, et al. Topology of the human and mouse m6A RNA methylomes revealed by m6A-seq. Nature. 2012:485(7397):201-6. 
42. Jia G, Fu Y, Zhao X, Dai Q, Zheng G, Yang Y, et al. N6-methyladenosine in nuclear RNA is a major substrate of the obesity-associated FTO. Nat Chem Biol. 2011;7(12):885-7.

43. Zhuang M, Li X, Zhu J, Zhang J, Niu F, Liang F, et al. The m6A reader YTHDF1 regulates axon guidance through translational control of Robo3.1 expression. Nucleic Acids Res. 2019;47(9):4765-77.

44. Wang X, Sun B, Jiang Q, Wu R, Cai M, Yao Y, et al. mRNA m(6)A plays opposite role in regulating UCP2 and PNPLA2 protein expression in adipocytes. Int J Obes (Lond). 2018;42(11):1912-24.

45. Dominissini D, Moshitch-Moshkovitz S, Salmon-Divon M, Amariglio N, Rechavi G. Transcriptome-wide mapping of N(6)-methyladenosine by m(6)A-seq based on immunocapturing and massively parallel sequencing. Nat Protoc. 2013;8(1):176-89.

46. Chen K, Lu Z, Wang X, Fu Y, Luo GZ, Liu N, et al. High-resolution N(6) -methyladenosine (m(6) A) map using photo-crosslinking-assisted $m(6)$ A sequencing. Angew Chem Int Ed Engl. 2015;54(5):1587-90.

47. Konno M, Koseki J, Asai A, Yamagata A, Shimamura T, Motooka D, et al. Distinct methylation levels of mature microRNAs in gastrointestinal cancers. Nat Commun. 2019;10(1):3888.

48. Bokar JA, Rath-Shambaugh ME, Ludwiczak R, Narayan P, Rottman F. Characterization and partial purification of mRNA N6-adenosine methyltransferase from HeLa cell nuclei. Internal mRNA methylation requires a multisubunit complex. J Biol Chem. 1994;269(26):17697-704.

49. Wang P, Doxtader KA, Nam Y. Structural basis for cooperative function of Mettl3 and Mettl14 methyltransferases. Mol Cell. 2016:63(2):306-17.

50. Wang X, Feng J, Xue Y, Guan Z, Zhang D, Liu Z, et al. Structural basis of N(6)-adenosine methylation by the METTL3-METTL14 complex. Nature. 2016;534(7608):575-8

51. Zhou Kl, Pan T. Structures of the m(6)A methyltransferase complex: two subunits with distinct but coordinated roles. Mol Cell. 2016;63(2):183-5.

52. Ping $X L$, Sun BF, Wang $L$, Xiao W, Yang $X$, Wang WJ, et al. Mammalian WTAP is a regulatory subunit of the RNA N6-methyladenosine methyltransferase. Cell Res. 2014;24(2):177-89.

53. Patil DP, Chen CK, Pickering BF, Chow A, Jackson C, Guttman M, et al. m(6)A RNA methylation promotes XIST-mediated transcriptional repression. Nature. 2016:537(7620):369-73.

54. Knuckles P, Lence T, Haussmann IU, Jacob D, Kreim N, Carl SH, et al. Zc3h13/Flacc is required for adenosine methylation by bridging the mRNA-binding factor Rbm15/Spenito to the m(6)A machinery component Wtap/FI(2)d. Genes Dev. 2018:32(5-6):415-29.

55. Yue Y, Liu J, Cui X, Cao J, Luo G, Zhang Z, et al. VIRMA mediates preferential m(6)A mRNA methylation in $3^{\prime} U T R$ and near stop codon and associates with alternative polyadenylation. Cell Discov. 2018;4:10.

56. Wen J, Lv R, Ma H, Shen H, He C, Wang J, et al. Zc3h13 regulates nuclear RNA m(6)A methylation and mouse embryonic stem cell self-renewal. Mol Cell. 2018;69(6):1028-38.e6.

57. Warda AS, Kretschmer J, Hackert P, Lenz C, Urlaub H, Höbartner C, et al. Human METTL16 is a N(6)-methyladenosine (m(6)A) methyltransferase that targets pre-mRNAs and various non-coding RNAs. EMBO Rep. 2017;18(11):2004-14.

58. Xiao W, Adhikari S, Dahal U, Chen YS, Hao YJ, Sun BF, et al. Nuclear m(6)A reader YTHDC1 regulates mRNA splicing. Mol Cell. 2016;61(4):507-19.

59. Roundtree IA, Luo GZ, Zhang Z, Wang X, Zhou T, Cui Y, et al. YTHDC1 mediates nuclear export of N(6)-methyladenosine methylated mRNAs. Elife. 2017:6:e31311.

60. Lesbirel S, Viphakone N, Parker M, Parker J, Heath C, Sudbery I, et al. The m(6)A-methylase complex recruits TREX and regulates mRNA export. Sci Rep. 2018:8(1):13827.

61. Wojtas MN, Pandey RR, Mendel M, Homolka D, Sachidanandam R, Pillai RS. Regulation of m(6)A transcripts by the $3^{\prime} \rightarrow 5^{\prime}$ RNA helicase YTHDC2 is essential for a successful meiotic program in the mammalian germline. Mol Cell. 2017:68(2):374-87.e12.

62. Hsu PJ, Zhu Y, Ma H, Guo Y, Shi X, Liu Y, et al. Ythdc2 is an N(6)-methyladenosine binding protein that regulates mammalian spermatogenesis. Cell Res. 2017;27(9):1115-27.

63. Du H, Zhao Y, He J, Zhang Y, Xi H, Liu M, et al. YTHDF2 destabilizes m(6) A-containing RNA through direct recruitment of the CCR4-NOT deadenylase complex. Nat Commun. 2016;7:12626.

64. Wang X, Zhao BS, Roundtree IA, Lu Z, Han D, Ma H, et al. N(6)-methyladenosine modulates messenger RNA translation efficiency. Cell. 2015:161(6):1388-99.
65. Li A, Chen YS, Ping XL, Yang X, Xiao W, Yang Y, et al. Cytoplasmic m(6)A reader YTHDF3 promotes mRNA translation. Cell Res. 2017;27(3):444-7.

66. Shi H, Wang X, Lu Z, Zhao BS, Ma H, Hsu PJ, et al. YTHDF3 facilitates translation and decay of N(6)-methyladenosine-modified RNA. Cell Res. 2017;27(3):315-28.

67. Huang H, Weng $H$, Sun W, Qin X, Shi H, Wu H, et al. Recognition of RNA N(6)-methyladenosine by IGF2BP proteins enhances mRNA stability and translation. Nat Cell Biol. 2018;20(3):285-95.

68. Meyer KD, Patil DP, Zhou J, Zinoviev A, Skabkin MA, Elemento O, et al. 5' UTR m(6)A promotes cap-independent translation. Cell. 2015;163(4):999-1010.

69. Alarcón CR, Goodarzi H, Lee H, Liu X, Tavazoie S, Tavazoie SF. HNRNPA2B1 is a mediator of $\mathrm{m}(6) \mathrm{A}$-dependent nuclear RNA processing events. Cell. 2015;162(6):1299-308.

70. Liu N, Dai Q, Zheng G, He C, Parisien M, Pan T. N(6)-methyladenosinedependent RNA structural switches regulate RNA-protein interactions. Nature. 2015;518(7540):560-4

71. Liu N, Zhou Kl, Parisien M, Dai Q, Diatchenko L, Pan T. N6-methyladenosine alters RNA structure to regulate binding of a low-complexity protein. Nucleic Acids Res. 2017;45(10):6051-63.

72. Zheng G, Dahl JA, Niu Y, Fedorcsak P, Huang CM, Li CJ, et al. ALKBH5 is a mammalian RNA demethylase that impacts RNA metabolism and mouse fertility. Mol Cell. 2013;49(1):18-29.

73. Ueda Y, Ooshio I, Fusamae Y, Kitae K, Kawaguchi M, Jingushi K, et al. AlkB homolog 3-mediated tRNA demethylation promotes protein synthesis in cancer cells. Sci Rep. 2017;7:42271.

74. Shi H, Wei J, He C. Where, when, and how: context-dependent functions of RNA methylation writers, readers, and erasers. Mol Cell. 2019;74(4):640-50.

75. Yu J, Chen M, Huang H, Zhu J, Song H, Zhu J, et al. Dynamic m6A modification regulates local translation of mRNA in axons. Nucleic Acids Res. 2018:46(3):1412-23.

76. Berulava T, Buchholz E, Elerdashvili V, Pena T, Islam MR, Lbik D, et al. Changes in m6A RNA methylation contribute to heart failure progression by modulating translation. Eur J Heart Fail. 2020;22(1):54-66.

77. Sun T, Wu R, Ming L. The role of m6A RNA methylation in cancer. Biomed Pharmacother. 2019:112:108613.

78. Alarcón CR, Lee H, Goodarzi H, Halberg N, Tavazoie SF. N6-methyladenosine marks primary microRNAs for processing. Nature. 2015;519(7544):482-5.

79. Ma JZ, Yang F, Zhou CC, Liu F, Yuan JH, Wang F, et al. METTL14 suppresses the metastatic potential of hepatocellular carcinoma by modulating N(6) -methyladenosine-dependent primary MicroRNA processing. Hepatology. 2017;65(2):529-43.

80. Zhang J, Bai R, Li M, Ye H, Wu C, Wang C, et al. Excessive miR-25-3p maturation via N(6)-methyladenosine stimulated by cigarette smoke promotes pancreatic cancer progression. Nat Commun. 2019;10(1):1858.

81. Park YM, Hwang SJ, Masuda K, Choi KM, Jeong MR, Nam DH, et al. Heterogeneous nuclear ribonucleoprotein C1/C2 controls the metastatic potential of glioblastoma by regulating PDCD4. Mol Cell Biol. 2012;32(20):4237-44.

82. Klinge CM, Piell KM, Tooley CS, Rouchka EC. HNRNPA2/B1 is upregulated in endocrine-resistant LCC9 breast cancer cells and alters the miRNA transcriptome when overexpressed in MCF-7 cells. Sci Rep. 2019;9(1):9430.

83. Berulava T, Rahmann S, Rademacher K, Klein-Hitpass L, Horsthemke B. N6-adenosine methylation in MiRNAs. PLoS One. 2015;10(2):e0118438.

84. Yang L, Ma Y, Han W, Li W, Cui L, Zhao X, et al. Proteinase-activated receptor 2 promotes cancer cell migration through RNA methylationmediated repression of miR-125b. J Biol Chem. 2015;290(44):26627-37.

85. Chen RX, Chen X, Xia LP, Zhang JX, Pan ZZ, Ma XD, et al. N(6)-methyladenosine modification of circNSUN2 facilitates cytoplasmic export and stabilizes HMGA2 to promote colorectal liver metastasis. Nat Commun. 2019;10(1):4695.

86. Yang $Y$, Fan X, Mao M, Song X, Wu P, Zhang Y, et al. Extensive translation of circular RNAs driven by N(6)-methyladenosine. Cell Res. 2017;27(5):626-41.

87. Park OH, Ha H, Lee Y, Boo SH, Kwon DH, Song HK, et al. Endoribonucleolytic cleavage of m(6)A-containing RNAs by RNase P/MRP complex. Mol Cell. 2019;74(3):494-507.e8.

88. Chen YG, Chen R, Ahmad S, Verma R, Kasturi SP, Amaya L, et al. N6-Methyladenosine modification controls circular RNA immunity. Mol Cell. 2019:76(1):96-109.e9. 
89. Chen X, Chen Z, Yu S, Nie F, Yan S, Ma P, et al. Long noncoding RNA LINC01234 functions as a competing endogenous RNA to regulate CBFB expression by sponging miR-204-5p in gastric cancer. Clin Cancer Res. 2018;24(8):2002-14.

90. Mendel M, Chen KM, Homolka D, Gos P, Pandey RR, McCarthy $A A$, et al. Methylation of structured RNA by the m(6)A writer METTL16 is essential for mouse embryonic development. Mol Cell. 2018;71(6):986-1000.e11.

91. Ma H, Wang X, Cai J, Dai Q, Natchiar SK, Lv R, et al. N(6-)Methyladenosine methyltransferase ZCCHC4 mediates ribosomal RNA methylation. Nat Chem Biol. 2019;15(1):88-94

92. Chen J, Wang C, Fei W, Fang X, Hu X. Epitranscriptomic m6A modification in the stem cell field and its effects on cell death and survival. Am J Cancer Res. 2019;9(4):752-64.

93. Zhou X, Han J, Zhen X, Liu Y, Cui Z, Yue Z, et al. Analysis of genetic alteration signatures and prognostic values of $\mathrm{m} 6 \mathrm{~A}$ regulatory genes in head and neck squamous cell carcinoma. Front Oncol. 2020;10:718.

94. Sun T, Wu Z, Wang X, Wang Y, Hu X, Qin W, et al. LNC942 promoting METTL14-mediated $m(6) A$ methylation in breast cancer cell proliferation and progression. Oncogene. 2020;39(31):5358-72.

95. Niu Y, Lin Z, Wan A, Chen H, Liang H, Sun L, et al. RNA N6-methyladenosine demethylase FTO promotes breast tumor progression through inhibiting BNIP3. Mol Cancer. 2019;18(1):46.

96. Zhou P, Wu M, Ye C, Xu Q, Wang L. Meclofenamic acid promotes cisplatin-induced acute kidney injury by inhibiting fat mass and obesity-associated protein-mediated m(6)A abrogation in RNA. J Biol Chem. 2019;294(45):16908-17.

97. Song H, Feng $X$, Zhang H, Luo Y, Huang J, Lin M, et al. METTL3 and ALKBH5 oppositely regulate $\mathrm{m}(6) \mathrm{A}$ modification of TFEB mRNA, which dictates the fate of hypoxia/reoxygenation-treated cardiomyocytes. Autophagy. 2019;15(8):1419-37.

98. Zhang P, He Q, Lei Y, Li Y, Wen X, Hong M, et al. m(6)A-mediated ZNF750 repression facilitates nasopharyngeal carcinoma progression. Cell Death Dis. 2018;9(12):1169.

99. Jin S, Zhang X, Miao Y, Liang P, Zhu K, She Y, et al. m(6)A RNA modification controls autophagy through upregulating ULK1 protein abundance. Cell Res. 2018;28(9):955-7.

100. Wang X, Wu R, Liu Y, Zhao Y, Bi Z, Yao Y, et al. m(6)A mRNA methylation controls autophagy and adipogenesis by targeting Atg5 and Atg7. Autophagy. 2020;16(7):1221-35.

101. Cui YH, Yang S, Wei J, Shea CR, Zhong W, Wang F, et al. Autophagy of the m(6)A mRNA demethylase FTO is impaired by low-level arsenic exposure to promote tumorigenesis. Nat Commun. 2021;12(1):2183.

102. Chen Y, Wang J, Xu D, Xiang Z, Ding J, Yang X, et al. m(6)A mRNA methylation regulates testosterone synthesis through modulating autophagy in Leydig cells. Autophagy. 2021;17(2):457-75.

103. Yang $S$, Wei J, Cui YH, Park G, Shah P, Deng Y, et al. m(6)A mRNA demethylase FTO regulates melanoma tumorigenicity and response to anti-PD-1 blockade. Nat Commun. 2019;10(1):2782.

104. Diao MY, Zhu Y, Yang J, Xi SS, Wen X, Gu Q, et al. Hypothermia protects neurons against ischemia/reperfusion-induced pyroptosis via m6Amediated activation of PTEN and the PI3K/Akt/GSK-3 $\beta$ signaling pathway. Brain Res Bull. 2020;159:25-31.

105. Zha X, Xi X, Fan X, Ma M, Zhang Y, Yang Y. Overexpression of METTL3 attenuates high-glucose induced RPE cell pyroptosis by regulating miR25-3p/PTEN/Akt signaling cascade through DGCR8. Aging (Albany NY). 2020;12(9):8137-50

106. Guo M, Yan R, Ji Q, Yao H, Sun M, Duan L, et al. IFN regulatory Factor-1 induced macrophage pyroptosis by modulating m6A modification of circ_0029589 in patients with acute coronary syndrome. Int Immunopharmacol. 2020;86:106800.

107. Mou Y, Wang J, Wu J, He D, Zhang C, Duan C, et al. Ferroptosis, a new form of cell death: opportunities and challenges in cancer. J Hematol Oncol. 2019;12(1):34.

108. Song Z, Jia G, Ma P, Cang S. Exosomal miR-4443 promotes cisplatin resistance in non-small cell lung carcinoma by regulating FSP1 m6A modification-mediated ferroptosis. Life Sci. 2021;276:119399.

109. Ma L, Zhang X, Yu K, Xu X, Chen T, Shi Y, et al. Targeting SLC3A2 subunit of system $X(C)(-)$ is essential for $m(6)$ A reader YTHDC2 to be an endogenous ferroptosis inducer in lung adenocarcinoma. Free Radic Biol Med. 2021;168:25-43.
110. Sarcognato S, Jong IEM, Fabris L, Cadamuro M, Guido M. Necroptosis in cholangiocarcinoma Cells. 2020;9(4):982.

111. Lan H, Liu Y, Liu J, Wang X, Guan Z, Du J, et al. Tumor-associated macrophages promote oxaliplatin resistance via METTL3-mediated $\mathrm{m}(6) \mathrm{A}$ of TRAF5 and necroptosis in colorectal cancer. Mol Pharm. 2021;18(3):1026-37.

112. Gong Y, Fan Z, Luo G, Yang C, Huang Q, Fan K, et al. The role of necroptosis in cancer biology and therapy. Mol Cancer. 2019;18(1):100.

113. Li Q, Ni Y, Zhang L, Jiang $R$, Xu J, Yang $H$, et al. HIF-1a-induced expression of $\mathrm{m} 6 \mathrm{~A}$ reader YTHDF1 drives hypoxia-induced autophagy and malignancy of hepatocellular carcinoma by promoting ATG2A and ATG14 translation. Signal Transduct Target Ther. 2021;6(1):76.

114. Li B, Jiang J, Assaraf YG, Xiao H, Chen ZS, Huang C. Surmounting cancer drug resistance: new insights from the perspective of N(6)-methyladenosine RNA modification. Drug Resist Updat. 2020;53:100720.

115. Vu LP, Pickering BF, Cheng Y, Zaccara S, Nguyen D, Minuesa G, et al. The N(6)-methyladenosine (m(6)A)-forming enzyme METTL3 controls myeloid differentiation of normal hematopoietic and leukemia cells. Nat Med. 2017;23(11):1369-76.

116. Wang Q, Geng W, Guo H, Wang Z, Xu K, Chen C, et al. Emerging role of RNA methyltransferase METTL3 in gastrointestinal cancer. J Hematol Oncol. 2020;13(1):57.

117. Lin S, Liu J, Jiang W, Wang P, Sun C, Wang X, et al. METTL3 promotes the proliferation and mobility of gastric cancer cells. Open Med (Wars). 2019;14:25-31.

118. Liu S, Li Q, Li G, Zhang Q, Zhuo L, Han X, et al. The mechanism of m(6)A methyltransferase METTL3-mediated autophagy in reversing gefitinib resistance in NSCLC cells by $\beta$-elemene. Cell Death Dis. 2020;11(11):969.

119. Lewinska A, Adamczyk-Grochala J, Deregowska A, Wnuk M. Sulforaphane-induced cell cycle arrest and senescence are accompanied by DNA Hypomethylation and changes in microRNA profile in breast cancer cells. Theranostics. 2017;7(14):3461-77.

120. Lin Z, Niu Y, Wan A, Chen D, Liang H, Chen X, et al. RNA m(6) A methylation regulates sorafenib resistance in liver cancer through FOXO3mediated autophagy. EMBO J. 2020;39(12):e103181.

121. Guo J, Wu Y, Du J, Yang L, Chen W, Gong K, et al. Deregulation of UBE2C-mediated autophagy repression aggravates NSCLC progression. Oncogenesis. 2018;7(6):49.

122. Huang $\mathrm{H}$, Weng $\mathrm{H}$, Chen J. m(6)A modification in coding and noncoding RNAs: roles and therapeutic implications in cancer. Cancer Cell. 2020;37(3):270-88.

123. Liu J, Ren D, Du Z, Wang H, Zhang H, Jin Y. m(6)A demethylase FTO facilitates tumor progression in lung squamous cell carcinoma by regulating MZF1 expression. Biochem Biophys Res Commun. 2018;502(4):456-64.

124. He H, Wu W, Sun Z, Chai L. MiR-4429 prevented gastric cancer progression through targeting METTL3 to inhibit m(6)A-caused stabilization of SEC62. Biochem Biophys Res Commun. 2019;517(4):581-7.

125. $L i \mathrm{~J}$, Xie H, Ying $Y$, Chen $\mathrm{H}$, Yan $\mathrm{H}$, He L, et al. YTHDF2 mediates the mRNA degradation of the tumor suppressors to induce AKT phosphorylation in N6-methyladenosine-dependent way in prostate cancer. Mol Cancer. 2020;19(1):152

126. Kleemann M, Schneider H, Unger K, Sander P, Schneider EM, FischerPosovszky $\mathrm{P}$, et al. MiR-744-5p inducing cell death by directly targeting HNRNPC and NFIX in ovarian cancer cells. Sci Rep. 2018;8(1):9020.

127. Zhu L, Zhu Y, Han S, Chen M, Song P, Dai D, et al. Impaired autophagic degradation of IncRNA ARHGAP5-AS1 promotes chemoresistance in gastric cancer. Cell Death Dis. 2019;10(6):383.

128. Taketo K, Konno M, Asai A, Koseki J, Toratani M, Satoh T, et al. The epitranscriptome $\mathrm{m} 6 \mathrm{~A}$ writer METTL3 promotes chemo- and radioresistance in pancreatic cancer cells. Int J Oncol. 2018;52(2):621-9.

129. Peng L, Yuan X, Jiang B, Tang Z, Li GC. LncRNAs: key players and novel insights into cervical cancer. Tumour Biol. 2016;37(3):2779-88.

130. Naren D, Yan T, Gong Y, Huang J, Zhang D, Sang L, et al. High Wilms' tumor 1 associating protein expression predicts poor prognosis in acute myeloid leukemia and regulates m(6)A methylation of MYC mRNA. J Cancer Res Clin Oncol. 2021;147(1):33-47.

131. Ma H, Shen L, Yang H, Gong H, Du X, Li J. m6A methyltransferase Wilms' tumor 1 -associated protein facilitates cell proliferation and cisplatin resistance in NKJT cell lymphoma by regulating dual-specificity phosphatases 6 expression via m6A RNA methylation. IUBMB Life. 2021;73(1):108-17. 
132. Paris J, Morgan M, Campos J, Spencer GJ, Shmakova A, Ivanova I, et al. Targeting the RNA m(6)A reader YTHDF2 selectively compromises cancer stem cells in acute myeloid leukemia. Cell Stem Cell. 2019;25(1):137-48.e6.

133. Yuan Y, Yan G, He M, Lei H, Li L, Wang Y, et al. ALKBH5 suppresses tumor progression via an $\mathrm{m}(6) \mathrm{A}$-dependent epigenetic silencing of pre-miR-181b-1/YAP signaling axis in osteosarcoma. Cell Death Dis. 2021;12(1):60.

134. Guo X, Li K, Jiang W, Hu Y, Xiao W, Huang Y, et al. RNA demethylase ALKBH5 prevents pancreatic cancer progression by posttranscriptional activation of PER1 in an m6A-YTHDF2-dependent manner. Mol Cancer. 2020;19(1):91

135. Zhang Z, Zhu H, Hu J. CircRAB1 1FIP1 promoted autophagy flux of ovarian cancer through DSC1 and miR-129. Cell Death Dis. 2021;12(2):219.

136. Su R, Dong L, Li C, Nachtergaele S, Wunderlich M, Qing Y, et al. R-2HG exhibits anti-tumor activity by targeting $\mathrm{FTO} / \mathrm{m}(6) \mathrm{A} / \mathrm{MYC} / \mathrm{CEBPA}$ signaling. Cell. 2018;172(1-2):90-105.e23.

137. Sun K, Du Y, Hou Y, Zhao M, Li J, Du Y, et al. Saikosaponin D exhibits anti-leukemic activity by targeting $\mathrm{FTO} / \mathrm{m}(6)$ A signaling. Theranostics. 2021;11(12):5831-46.

138. Zhang R, Li SW, Liu L, Yang J, Huang G, Sang Y. TRIM11 facilitates chemoresistance in nasopharyngeal carcinoma by activating the $\beta$-catenin/ ABCC9 axis via p62-selective autophagic degradation of Daple. Oncogenesis. 2020;9(5):45.

139. Wang $X$, Tian L, Li Y, Wang J, Yan B, Yang L, et al. RBM15 facilitates laryngeal squamous cell carcinoma progression by regulating TMBIM6 stability through IGF2BP3 dependent. J Exp Clin Cancer Res. 2021;40(1):80.

140. Chen H, Yao J, Bao R, Dong Y, Zhang T, Du Y, et al. Cross-talk of four types of RNA modification writers defines tumor microenvironment and pharmacogenomic landscape in colorectal cancer. Mol Cancer. 2021;20(1):29.

141. Chen H, Gao S, Liu W, Wong CC, Wu J, Wu J, et al. RNA N(6)-Methyladenosine methyltransferase METTL3 facilitates colorectal cancer by activating the $\mathrm{m}(6) \mathrm{A}-\mathrm{GLUT1}$-mTORC1 axis and is a therapeutic target. Gastroenterology. 2021;160(4):1284-300.e16.

142. Li N, Kang Y, Wang L, Huff S, Tang R, Hui H, et al. ALKBH5 regulates antiPD-1 therapy response by modulating lactate and suppressive immune cell accumulation in tumor microenvironment. Proc Natl Acad Sci U S A. 2020;117(33):20159-70.

143. Zhou Z, LV J, Yu H, Han J, Yang X, Feng D, et al. Mechanism of RNA modification N6-methyladenosine in human cancer. Mol Cancer. 2020;19(1):104

144. Lee YS, Lee DH, Choudry HA, Bartlett DL, Lee YJ. Ferroptosis-induced endoplasmic reticulum stress: cross-talk between ferroptosis and apoptosis. Mol Cancer Res. 2018;16(7):1073-6.

145. Buccarelli M, Marconi M, Pacioni S, De Pascalis I, D’Alessandris QG, Martini M, et al. Inhibition of autophagy increases susceptibility of glioblastoma stem cells to temozolomide by igniting ferroptosis. Cell Death Dis. 2018;9(8):841.

146. Yang J, Liu J, Zhao S, Tian F. N(6)-Methyladenosine METTL3 modulates the proliferation and apoptosis of Lens epithelial cells in diabetic cataract. Mol Ther Nucleic Acids. 2020;20:111-6.

147. Ma H, Shen L, Yang H, Gong H, Du X, Li J. m6A methyltransferase Wilms' tumor 1-associated protein facilitates cell proliferation and cisplatin resistance in NK/T cell lymphoma by regulating dual-specificity phosphatases 6 expression via m6A RNA methylation. IUBMB Life. 2021;73(1):108-17.

\section{Publisher's Note}

Springer Nature remains neutral with regard to jurisdictional claims in published maps and institutional affiliations.

Ready to submit your research? Choose BMC and benefit from:

- fast, convenient online submission

- thorough peer review by experienced researchers in your field

- rapid publication on acceptance

- support for research data, including large and complex data types

- gold Open Access which fosters wider collaboration and increased citations

- maximum visibility for your research: over 100M website views per year

At BMC, research is always in progress.

Learn more biomedcentral.com/submissions 